Título artículo / Títol article: Manufacturing variation models in multi-station machining systems

Autores / Autors

José Vicente Abellán-Nebot, Fernando Romero Subirón, Julio Serrano Mira

Revista:

The International Journal of Advanced Manufacturing Technology

Versión / Versió:

Versió post-print

Cita bibliográfica / Cita bibliogràfica (ISO 690):
ABELLÁN-NEBOT, José Vicente; SUBIRÓN, Fernando Romero; MIRA, Julio Serrano. Manufacturing variation models in multi-station machining systems. The International Journal of Advanced Manufacturing Technology, 2013, vol. 64, no 1-4, p. 63-83.

url Repositori UJI: 


\title{
Manufacturing variation models in multi-station machining systems
}

\author{
José V. Abellan-Nebot • Fernando Romero Subirón • Julio Serrano Mira
}

Received: date / Accepted: date

\begin{abstract}
In product design and quality improvement fields, the development of reliable 3D machining variation models for multi-station machining processes is a key issue to estimate the resulting geometrical and dimensional quality of manufactured parts, generate robust process plans, eliminate downstream manufacturing problems and reduce ramp-up times. In the literature, two main 3D machining variation models have been studied: the Stream of Variation (SoV) model, oriented to product quality improvement (fault diagnosis, process planning evaluation and selection, etc.), and the Model of the Manufactured Part (MoMP), oriented to product and manufacturing design activities (manufacturing and product tolerance analysis and synthesis). This paper reviews the fundamentals of each model and describes step by step how to derive them using a simple case study. The paper analyzes both models and compares their main characteristics and applications. A discussion about the drawbacks and limitations of each model and some potential research lines in this field are also presented.
\end{abstract}

Keywords Multi-station Machining Processes · Part Quality · SoV · MoMP · Manufacturing Variation Model

J.V. Abellan-Nebot

Department of Industrial Systems Engineering and Design

Universitat Jaume I, Castellon de la Plana, Spain

Tel.: +34-964-728186

Fax: +34-964-728170

E-mail: abellan@esid.uji.es

F. Romero Subirón

E-mail: fromero@esid.uji.es

J. Serrano Mira

E-mail: jserrano@esid.uji.es

\section{Nomenclature}

$C S: \quad$ Coordinate system.

$R: \quad$ Reference CS.

$F_{k}: \quad$ Fixture CS at station $k$.

$H_{k, j}$ : $\quad$ CS of $j$ th part-holder surface at station $k$.

$M_{k}$ : Machine-tool CS at station $k$.

$M_{k, o}$ : $\quad$ CS of the oth machining operation at station $k$.

$M_{k, o_{i}}$ : CS of the cutting-tool tip when machining surface $S_{i}$.

$G$ : $\quad$ Gauge CS.

$G_{p}$ : $\quad$ CS of the $p$ th positioning gauge surface.

$S_{l}$ : $\quad$ CS of the $l$ th locating datum surface.

$S_{i}: \quad$ CS of the $i$ th machined surface.

$S_{m}$ : $\quad$ CS of the $m$ th measurement datum surface.

$S_{q}$ : $\quad$ CS of the $q$ th inspected part surface or toleranced surface.

$\mathbf{d}_{F}^{R}: \quad \quad \quad$ Small translational deviations of $\mathrm{F}$ with respect to (w.r.t.) R, $\left[d_{F x}^{R}, d_{F y}^{R}, d_{F z}^{R}\right]^{T}$.

$\boldsymbol{\theta}_{F}^{R}$ : $\quad$ Small orientational deviations of $\mathrm{F}$ w.r.t. $\mathrm{R},\left[\theta_{F x}^{R}, \theta_{F y}^{R}, \theta_{F z}^{R}\right]^{T}$.

$\mathbf{x}_{F}^{R}$ : $\quad$ Differential motion vector (DMV) of $\mathrm{F}$ w.r.t. $\mathrm{R},\left[\left(\mathbf{d}_{F}^{R}\right)^{T},\left(\boldsymbol{\theta}_{F}^{R}\right)^{T}\right]^{T}$.

$\left\{\mathbf{T}_{R, F}\right\}_{G}$ : Small displacement torsor (SDT) of F at $\mathrm{R}$ expressed in the $\mathrm{G}$ coordinate system, $\left\{\mathbf{T}_{R, F}\right\}_{G}=\left\{\begin{array}{l}\boldsymbol{\Omega}_{R, F} \\ \mathbf{D}_{R, F}\end{array}\right\}$.

$\boldsymbol{\Omega}_{R, F} \quad$ Orientation deviations of the SDT of $\mathrm{F}$ at $\mathrm{R},[\alpha, \beta, \gamma]^{T}$.

$\mathbf{D}_{R, F} \quad$ Translational deviations of the SDT of $\mathrm{F}$ at $\mathrm{R},[u, v, w]^{T}$.

$\mathbf{t}_{F}^{R}: \quad \quad$ Position vector of $F$ w.r.t. $R$.

$\varphi_{F}^{R}: \quad \quad$ Orientation vector of $F$ w.r.t. $R$. 


\section{Introduction}

Traditionally, product design has been separated from manufacturing process design along the product development cycle increasing ramp-up times, product changes costs and variability of product quality. This productoriented approach, named as over-the-wall design due to the sequential nature of the design activities, prevents the integration of design and manufacturing activities to improve product development [1]. In order to overcome this limitation, manufacturers have begun to investigate ways to simultaneously evaluate product designs and manufacturing processes in an attempt to eliminate downstream manufacturing problems and reduce ramp-up times. For this purpose, product design requires the application of process-oriented approaches through 3D manufacturing variation models to integrate product and manufacturing process information [2] However, the application of $3 \mathrm{D}$ manufacturing variation models is nowadays limited, specially in multi-station machining processes (MMPs) where a large number of machining operations is conducted in different stations with different fixture devices. In fact, in product design and quality improvement fields, the development of reliable $3 \mathrm{D}$ variation models of MMPs is a key issue to estimate the resulting geometrical and dimensional quality of manufactured parts.

In the literature, one can distinguish two important group of researchers dealing with the development of $3 \mathrm{D}$ variation models for MMPs: i) a first group of researchers more focused on the quality improvement field, mostly universities from USA ii) and a second group of researchers focused on product design, mostly universities from France and Canada. The research conducted by the first group, that we name as "IO school" (Industrial Operations school) hereafter, is focused on modeling the dimensional and geometrical variation of manufactured parts by the state space model which is commonly applied in control theory [3]. Through this model, a large number of quality improvement activities can be conducted such as part quality control, optimal placement of inspection stations, robust process planning, process-oriented tolerancing, etc. The research conducted by the second group, that we name as "PD school" (Product Design school) hereafter, is focused on modeling dimensional and geometrical part quality variations considering the system workpiece/ fixture/ machine-tool as a mechanical assembly. By this consideration, well-known approaches for the analysis of mechanical assemblies can be applied. Specifically, the PD school applies the concept of small displacement torsors (SDTs) to describe and propagate surface devi- ations along different machining stations. The application of the PD school investigations are mainly focused on tolerance analysis and synthesis.

Both schools have been very active during last decade, and their 3D manufacturing variation models have been used in a large number of applications. Surprisingly enough, these schools have been working in parallel, without using potential synergies or adapting the advances from one school to the other. Moreover, despite the success of both schools and the large number of publications related to them, there is no review in the literature that analyzes and compares both 3D manufacturing variation models. This paper contributes to fill this literature gap, providing a critical comparison of both models, paying special attention to: i) fixtures and processes supported, ii) limitations of virtual inspection, iii) GD\&T conformance, iv) applicability, v) modeling accuracy, vi) modeling complexity. Furthermore, the review also exposes future lines of research that should be addressed by both schools. The paper is organized as follows. Section 2 describes the fundamentals of the 3D manufacturing variation model applied by the IO school and its main applications. Similarly, Section 3 describes the 3D manufacturing variation model applied by the PD school together with its main applications. Section 4 presents two modeling 2D examples (extensible to any 3D case study) to show, step by step, the implementation of both models, analyzing them symbolically and numerically. Section 5 discusses both $3 \mathrm{D}$ variation models, comparing their main drawbacks and advantages. Finally, Section 6 concludes the paper and outlines some potential research lines in the field.

\section{Variation modeling and propagation by the IO school: The Stream of Variation model}

\subsection{Fundamentals}

Manufacturing variability in MMPs and its impact on part quality can be modeled by capturing the mathematical relationship between the sources of variation of manufacturing process variables that are critical to part quality (named key control characteristics -KCCsof the process) and the deviations of the functional features of the product (named key product characteristics -KPCs-). This relationship is modeled through a non-linear function $y_{1}=f_{1}\left(u_{1}, u_{2}, \ldots, u_{n}\right)$, where $y_{1}$ is a KPC and $u_{1}, u_{2}, \ldots, u_{n}$ are the KCCs in the MMP. By the assumption of small variations, the non-linear 
model can be linearized through a Taylor series expansion and the part quality variation can be defined as

$$
\begin{aligned}
\Delta y_{1}= & \left.\frac{\delta f_{1}(\mathbf{u})}{\delta u_{1}}\right|_{\mathbf{u}=\overline{\mathbf{u}}} \cdot\left(u_{1}-\bar{u}_{1}\right)+\cdots+ \\
& +\left.\frac{\delta f_{1}(\mathbf{u})}{\delta u_{n}}\right|_{\mathbf{u}=\overline{\mathbf{u}}} \cdot\left(u_{n}-\bar{u}_{n}\right)+\varepsilon_{1},
\end{aligned}
$$

where $\mathbf{u}=\left[u_{1}, u_{2}, \ldots, u_{n}\right]^{T} ; \varepsilon_{1}$ contains the high-order non-linear residuals of the linearization, and the linearization point is defined by $\overline{\mathbf{u}}=\left[\bar{u}_{1}, \bar{u}_{2}, \ldots, \bar{u}_{n}\right]^{T}$. This linear approximation can be considered good enough for many MMPs [4]. Considering that there are $M$ KPCs in the part which are stacked in the vector $\mathbf{Y}=\left[\Delta y_{1}, \ldots\right.$, $\left.\Delta y_{M}\right]^{T}$, Eq. (1) can be re-written in a matrix form as

$\mathbf{Y}=\boldsymbol{\Gamma} \cdot \mathbf{U}+\varepsilon$,

where $\mathbf{U}=\left[\Delta u_{1}, \ldots, \Delta u_{n}\right]^{T}$ and $\Delta u_{j}=u_{j}-\bar{u}_{j}$ for $j=1, \ldots, n$ defines the small variations of the KCCs in a MMP; $\boldsymbol{\Gamma}$ is the matrix $\left[\left[\left.\frac{\delta f_{1}(\mathbf{u})}{\delta u_{1}}\right|_{\mathbf{u}=\overline{\mathbf{u}}}, \ldots,\left.\frac{\delta f_{1}(\mathbf{u})}{\delta u_{n}}\right|_{\mathbf{u}=\overline{\mathbf{u}}}\right]\right.$; $\left.\ldots ;\left[\left.\frac{\delta f_{M}(\mathbf{u})}{\delta u_{1}}\right|_{\mathbf{u}=\overline{\mathbf{u}}}, \ldots,\left.\frac{\delta f_{M}(\mathbf{u})}{\delta u_{n}}\right|_{\mathbf{u}=\overline{\mathbf{u}}}\right]\right] ;$ and $\varepsilon$ is the stacked vector of the high-order non-linear residuals.

In MMPs, the derivation of Eq. (2) is a challenging task. Researchers from USA universities have proposed the adoption of the well-known state space model from control theory [3] to represent mathematically the relationship between the sources of variation of a MMP and the deviation of the machined surfaces at each station, including how the deviation of previous machined surfaces influences at current station when these surfaces are used as locating datums. In this representation, dimensional deviations of part surfaces from nominal values are defined by $6 \times 1$ vectors named state vectors, in the form of $\mathbf{x}_{k, i}=\left[\left(\mathbf{d}_{i}^{\circ}\right)^{T},\left(\boldsymbol{\theta}_{i}^{\circ}\right)^{T}\right]^{T}$, where $\mathbf{d}_{i}^{\circ}{ }^{i}=\left[d_{i x}^{\circ}, d_{i y}{ }^{i}, d_{i z}^{\circ}\right]^{T}$ is the small translational deviation and $\boldsymbol{\theta}_{i}^{\circ}{ }^{i}=\left[\theta_{i x}^{\circ}, \theta_{i y}^{\circ}, \theta_{i z}^{\circ}\right]^{T}$ is the small orientation deviation of the local CS of the $i$ th part surface. The notation ${ }^{\circ} i$ refers to the nominal CS and $i$ to the current CS. The deviation of all part surfaces at the station $k$ are stacked in the state vector $\mathbf{x}_{k}=\left[\mathbf{x}_{k, 1}^{T}, \ldots, \mathbf{x}_{k, i}^{T}, \ldots\right]^{T}$. Note that each feature deviation, $\mathbf{x}_{k, i}$, is expressed in its own CS.

In a machining station, three main sources of variation can be distinguished: datum-induced deviations, fixture-induced deviations and machining-induced deviations. The state space model defines analytically how these three main sources of error influence on the final part quality deviation. To illustrate how these three main sources of variation influence on part quality, we consider an $N$-station machining process shown in Figure 1 and the $k$ th machining station with the workpiece and the fixture device shown in Figure 2. At this $k$ th

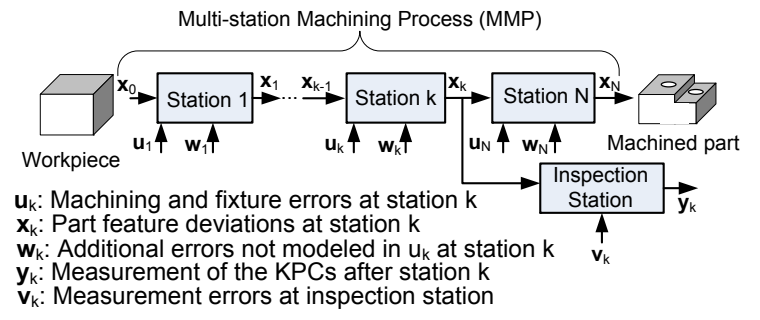

Fig. 1 Manufacturing variation propagation in a MMP

station, the following sources of variation exist.

First, the deviations of the datum surfaces used for locating the workpiece deviate the workpiece location from its nominal value. This term can be estimated as $\mathbf{x}_{k}^{d}=\mathbf{A}_{k} \cdot \mathbf{x}_{k-1}$, where $\mathbf{x}_{k-1}$ is the vector of part surface deviations from upstream machining stations and $\mathbf{A}_{k}$ linearly relates the datum deviations with the machined surface deviation due to the locating deviation of the workpiece.

Secondly, the fixture-induced deviations deviate the workpiece location on the machine-tool table and produce a machined surface deviation. This term can be estimated as $\mathbf{x}_{k}^{f}=\mathbf{B}_{k}^{f} \cdot \mathbf{u}_{k}^{f}$, where $\mathbf{u}_{k}^{f}$ is the vector of locator deviations and $\mathbf{B}_{k}^{f}$ is a matrix that linearly relates locator deviations with the machined surface deviation.

Thirdly, the operation or machining deviations such as those due to geometrical and kinematic errors, toolwear errors, etc., deviate the cutting-tool tip during machining and thus, the machined surface is deviated from its nominal value. This term is modeled as $\mathbf{x}_{k}^{m}=$ $\mathbf{B}_{k}^{m} \cdot \mathbf{u}_{k}^{m}$, where $\mathbf{u}_{k}^{m}$ is the vector that defines the KCCs related to operation or machining deviations and $\mathbf{B}_{k}^{m}$ is a matrix that linearly relates these KCCs with the machined surface deviations.

Therefore, for an $N$-station machining process the derivation of the state space model can be defined in a generic form as

$$
\mathbf{x}_{k}=\mathbf{A}_{k} \cdot \mathbf{x}_{k-1}+\mathbf{B}_{k} \cdot \mathbf{u}_{k}+\mathbf{w}_{k}, \quad k=1, \ldots, N,
$$

where $\mathbf{B}_{k} \cdot \mathbf{u}_{k}$ represents the deviations introduced within station $k$ due to the KCCs (related to fixturing and machining) and it is defined as $\left[\begin{array}{ll}\mathbf{B}_{k}^{f} & \mathbf{B}_{k}^{m}\end{array}\right] \cdot\left[\left(\mathbf{u}_{k}^{f}\right)^{T},\left(\mathbf{u}_{k}^{m}\right)^{T}\right]^{T}$; and $\mathbf{w}_{k}$ is the unmodeled system noise and linearization errors.

The derivation of the state space model in MMPs, named the stream of variation (SoV) model, was firstly presented by Huang et al. [5]. Djurjanovic et al. [6] expanded Huang's work in order to explicitly derive the 


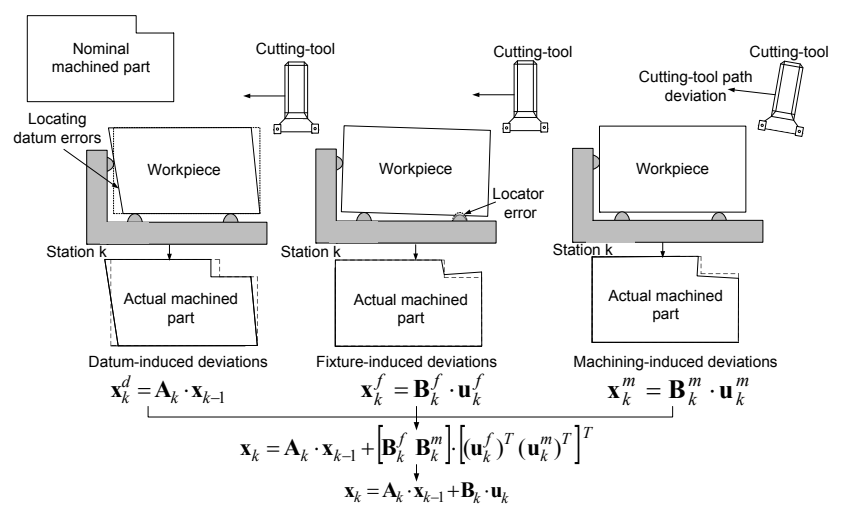

Fig. 2 Sources of variation and state space model formulation for station $k$

linear equations that model the relationships between fixtures, locating datum and measurement datum features. In their research work, a complex mathematical derivation was required and the methodology proposed was not straightforward to be applied. The SoV model derivation was improved by [4] who applied the DMV concept from robotics to represent the geometric deviation of each machined feature. In their work, a stepby-step methodology was proposed in order to derive the matrices $\mathbf{A}_{k}$ and $\mathbf{B}_{k}$ at each station using product and process information (i.e. part geometry and fixture layouts). Previous works were limited to 3-2-1 orthogonal fixture layouts based on locators and generic cutting-tool path deviations without explicitly including machining-induced errors. Loose et al. [7] extended the state space model formulation by including general non-orthogonal fixture layouts based on locators.More recently, Abellan-Nebot et al. [8] expanded the formulation of matrix $\mathbf{B}_{k}$ in order to include common machining sources of variation such as those due to tool wear, thermal expansions, cutting-tool deflections and geometric-kinematic machine-tool errors.

\subsection{Virtual part quality inspection and verification}

Somewhere along the MMP, an inspection station can be placed in order to inspect the KPCs and verify whether the workpiece/part is within specifications. Following the state space model formulation from control theory [3], a virtual inspection after the $k$ th machining station can be conducted using the expression:

$$
\mathbf{y}_{k}=\mathbf{C}_{k} \cdot \mathbf{x}_{k}+\mathbf{v}_{k}
$$

where $\mathbf{y}_{k}$ represents the deviations of the inspected $\mathrm{KPCs} ; \mathbf{C}_{k} \cdot \mathbf{x}_{k}$ are the deviations of the KPCs that are defined as a linear combination of the deviations of workpiece features at the $k$ th station; and $\mathbf{v}_{k}$ is the measurement noise of the inspection process. In a similar way to $\mathbf{x}_{k}$, vector $\mathbf{y}_{k}$ is defined as $\left[\mathbf{y}_{k, 1}^{T}, \ldots, \mathbf{y}_{k, q}^{T}, \ldots, \mathbf{y}_{k, M}^{T}\right]^{T}$, where $\mathbf{y}_{k, q}$ is the inspected deviation of the $q$ th KPC (denoted as $S_{q}$ ) defined by the vector $\mathbf{y}_{k, q}=\left[\left(\mathbf{d}_{S_{q}}^{S_{m}}\right)^{T},\left(\boldsymbol{\theta}_{S_{q}}^{S_{m}}\right)^{T}\right]^{T}$, where $S_{m}$ is the measurement datum surface and $M$ is the number of KPCs inspected. In Eq. (4), matrix $\mathbf{C}_{k}$ depends on what KPCs they are and which measurement datums are used to locate the part in the inspection station. How to derive matrix $\mathbf{C}_{k}$ is explained in detail in [4].

In order to express the part quality measurements by an explicit linear function of the KCCs presented along the MMP and considering that the inspection station is placed at the end of the MMP (after machining station $N$ ), Eqs. (3) and (4) can be combined and rewritten in the input-output form as:

$\mathbf{Y}=\boldsymbol{\Gamma} \cdot \mathbf{U}+\varepsilon$,

where the vectors $\mathbf{Y}$ and $\mathbf{U}$ are the stacking quality vectors after inspection and the vectors of sources of error respectively from the stations $k=1,2, \ldots, N$. In Eq. (5), vector $\mathbf{Y}$ is defined as $\mathbf{Y}=\left[\mathbf{y}_{N, 1}^{T}, \mathbf{y}_{N, 2}^{T}, \ldots, \mathbf{y}_{N, M}^{T}\right]^{T}$, vector $\mathbf{U}$ is defined as $\mathbf{U}=\left[\mathbf{u}_{1}^{T}, \mathbf{u}_{2}^{T}, \ldots, \mathbf{u}_{N}^{T}\right]^{T}$, and matrices $\boldsymbol{\Gamma}$ and $\varepsilon$ are defined as:

$$
\begin{aligned}
& \boldsymbol{\Gamma}=\left[\mathbf{M}_{N, 1}, \ldots, \mathbf{M}_{N, N}\right], \\
& \boldsymbol{\varepsilon}=\left[\overline{\mathbf{M}}_{N, 1}, \ldots, \overline{\mathbf{M}}_{N, N}\right] \cdot\left[\mathbf{w}_{1}, \ldots, \mathbf{w}_{N}\right]^{T}+\mathbf{v}_{N},
\end{aligned}
$$

where

$$
\begin{aligned}
& \mathbf{M}_{N, j}=\mathbf{C}_{N} \cdot \mathbf{\Phi}_{N, j} \cdot \mathbf{B}_{j}, \quad j \leq N, \\
& \overline{\mathbf{M}}_{N, j}=\mathbf{C}_{N} \cdot \mathbf{\Phi}_{N, j}, \\
& \boldsymbol{\Phi}_{N, j}=\left\{\begin{array}{lll}
\mathbf{A}_{N-1} \cdot \mathbf{A}_{N-2} \cdots \mathbf{A}_{j}, & \text { if } & j<N \\
\mathbf{I}, & \text { if } & j=N
\end{array}\right.
\end{aligned}
$$

Using Eq. (5), the deviation of the local CS of an inspected KPC $S_{q}$ w.r.t. the measurement datum $S_{m}$ can be estimated. However, if one wants to verify if an inspected KPC is within its tolerance zone according to geometric dimensioning and tolerancing (GD\&T) practices, the deviation of the boundary points of the inspected KPC w.r.t. the measurement datum should be evaluated. For this purpose, the deviation of the $r$ th boundary point $P_{r}$ of the inspected $q$ th KPC w.r.t. $S_{m}$ can be evaluated according to the expression:

$\mathbf{y}_{N, P_{r}}=\left(\begin{array}{cc}\mathbf{I}_{3 \times 3} & -\left(\hat{\mathbf{t}}_{P_{r}}^{S_{q}}\right) \\ \mathbf{0}_{3 \times 3} & \mathbf{I}_{3 \times 3}\end{array}\right) \cdot \mathbf{y}_{N, S_{q}}$,

where $\mathbf{y}_{N, S_{q}}$ is the deviation of the $q$ th KPC obtained from Eq. (5); $\mathbf{I}_{3 \times 3}$ is the $3 \times 3$ identity matrix; $\mathbf{0}_{3 \times 3}$ is the $3 \times 3$ zero matrix; and $\hat{\mathbf{t}}_{P_{r}}^{S_{q}}$ is the skew matrix of the 


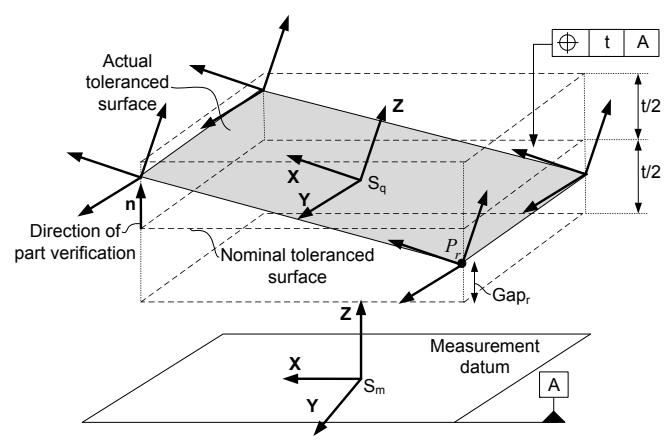

Fig. 3 Gap distance of a boundary point in a deviated toleranced surface.

nominal position vector $\mathbf{t}_{P_{r}}^{S_{q}}$ which describes the position of the point $P_{r}$ w.r.t. the $S_{q}$. The resulting deviation of point $P_{r}$ w.r.t. $S_{m}$ is then defined by a $6 \times 1$ deviation vector in the form of $\mathbf{y}_{N, P_{r}}=\left[\left(\mathbf{d}_{P_{r}}^{S_{m}}\right)^{T},\left(\boldsymbol{\theta}_{P_{r}}^{S_{m}}\right)^{T}\right]^{T}$. The deviation of the $r$ th point of the toleranced surface following the direction of part verification, defined by the vector $\mathbf{n}=\left[n_{x}, n_{y}, n_{z}\right]^{T}$, is evaluated by the expression:

$\left[\mathbf{y}_{N, P_{r}}\right]_{\mathbf{n}}=\mathbf{n}^{T} \cdot \mathbf{d}_{P_{r}}^{S_{m}}$

The tolerance zone where the variability of the manufactured feature will lie can be obtained by analyzing the deviation from nominal values of all boundary points of the KPC. If the tolerance of a KPC is defined from design specifications, then one can be interested in verifying the part according to a given MMP. For this purpose, the distance between each deviated boundary point and the specified tolerance zone from design should be evaluated. For the point $P_{r}$, this distance is defined as the gap distance Gap $_{r}$ and it is formulated as:

$\operatorname{Gap}_{r}=\min \left(\tau+\left[\mathbf{y}_{N, P_{r}}\right]_{\mathbf{n}}, \tau-\left[\mathbf{y}_{N, P_{r}}\right]_{\mathbf{n}}\right)$,

where $\tau$ is the maximum deviation of the $r$ th point according to the tolerance size (e.g. for a positional tolerance, $\tau$ is $t / 2$, where $t$ is the tolerance value). The $r$ th point of the inspected surface will be within the tolerance zone if Gap $_{r}$ remains positive or null (see Fig. 3). Analyzing the deviation of all boundary points of the $\mathrm{KPC}$, the verification of the GD\&T tolerances applied to the KPC can be conducted.

It should be remarked that the virtual inspection and verification can be conducted following two main approaches: the worst-case approach and the statistical approach. Depending on which type of approach is applied, the estimation of the KPC deviation, and thus, the estimation of the deviation of its boundary points in order to analyze a functional specification, will be more or less conservative. For each approach, the resulting estimation is derived as follows:

- Worst case approach: the estimated deviation of the $q$ th KPC will be the maximum according to the expected sources of variation. According to Eq. (5), the worst-case analysis can be conducted assuming that all coefficients from matrix $\boldsymbol{\Gamma}$ and vector $\mathbf{U}$ are positive and the measurement error also increases the expected deviation. The worst-case deviation of the $S_{q}$ CS is:

$\mathbf{y}_{N, S_{q-w c}}= \pm(|\boldsymbol{\Gamma}| \cdot|\mathbf{U}|+|\varepsilon|)$,

and the worst possible part quality considering the point boundary deviation is defined as:

$\operatorname{Gap}_{w c}=\min \left(\operatorname{Gap}_{r_{w c}}\right) \quad \forall r \in$ boundary,

where Gap $_{r_{w c}}$ is evaluated by Eqs. (10) and (12) considering $\mathbf{y}_{N, S_{q-w c}}$ instead of $\mathbf{y}_{N, S_{q}}$.

- Statistical approach: the worst-case analysis produces an estimation that is highly improbable, specially for a large number of sources of variation due to the randomness of the sources of variation in MMPs. To estimate a more probable scenario, the statistical analysis is commonly applied. In this analysis, the sources of variation are assumed to be independent to each other and normally distributed. Under these assumptions, the covariance of the $S_{q}$ CS can be estimated as:

$\boldsymbol{\Sigma}_{\mathbf{y}_{N, S_{q}}}=\boldsymbol{\Gamma} \cdot \boldsymbol{\Sigma}_{\mathbf{U}} \cdot \boldsymbol{\Gamma}^{T}+\boldsymbol{\Sigma}_{\boldsymbol{\varepsilon}}$

where $\boldsymbol{\Sigma}_{\boldsymbol{\bullet}}$ is the covariance matrix of $\bullet$. Therefore, the deviation of the KPC is estimated, according to $6 \sigma$, as:

$\mathbf{y}_{N, S_{q-s t}}= \pm 3 \cdot\left[\left(\boldsymbol{\Sigma}_{\mathbf{y}_{N, S_{q}}}(1,1)\right)^{1 / 2}, \ldots,\left(\boldsymbol{\Sigma}_{\mathbf{y}_{N, S_{q}}}(6,6)\right)^{1 / 2}\right]^{T}$

where $\boldsymbol{\Sigma}_{\bullet}(\varphi, \varphi)$ denotes the $(\varphi, \varphi)$ component of matrix $\Sigma_{\bullet}$. The part quality considering the point boundary deviations is defined as:

$\operatorname{Gap}_{s t}=\min \left(\operatorname{Gap}_{r_{s t}}\right) \quad \forall r \in$ boundary,

where Gap $_{r_{s t}}$ is evaluated by Eqs. (10) and (12) considering $\mathbf{y}_{N, S_{q-s t}}$ instead of $\mathbf{y}_{N, S_{q}}$.

\subsection{Main applications}

In the literature, the SoV model has been applied for a large number of applications such as part quality estimation and process planning [9-11], manufacturing fault identification [12-20], dimensional quality control [21-26] and process-oriented tolerancing [27,28]. 


\subsubsection{Part quality estimation and process planning}

The straightforward application of the SoV model is part quality estimation (i.e. tolerance analysis) which leads the designer to estimate if the MMP is able to manufacture parts within specifications. By this analysis, the process planner can search the most robust MMP to manufacturing disturbances from a group of candidates, and conduct specific modifications to improve the manufacturing process. Zhang et al. [9] presented a sensitivity analysis based on the SoV model to assess how sensitive the KPCs are to certain fixtureinduced variations in an MMP. Through the sensitivity indices, the robustness of each process plan candidate can be evaluated, and the critical stations and fixture components of each MMP can be detected and modified. Liu et al. [10] proposed a quality-assured setup planning to select the optimal process plan from a group of process plan candidates with different fixture layouts. The optimal process plan was referred to as the process plan candidate that minimizes the cost related to process precision and satisfies the quality specifications. Abellan-Nebot et al. [11] proposed the use of historical shop-floor quality data from existing MMPs to extract manufacturing operation capabilities in order to conduct a more accurate process planning. The process plan selection procedure was based on three components: i) inference on the process capabilities from shop floor data; ii) sensitivity analysis of candidate process plans to identify critical fixtures and manufacturing stations/operations; and iii) optimal selection of candidate process plans evaluating a multivariate capability ratio.

\subsubsection{Fault cause identification}

The issue of diagnosability refers to the problem of whether the measurements of the KPCs contain enough information for the diagnosis of critical process faults [14]. For instance, knowing the SoV model defined by Eq. (5) and measuring different KPCs (vector $\mathbf{Y}$ ), it may be possible to infer the sources of variation (vector $\mathbf{U}$ ). However, the MMPs are usually not diagnosable due to the inherent dimensional coupling between cutting-tool deviations and fixture deviations at each machining station. That is, fixture-induced deviations and machininginduced deviations may produce the same pattern deviation of KPCs. Consequently, it is difficult to distinguish error sources at each operation. To overcome this limitation, Wang et al. [12] applied the SoV model and proposed the equivalent fixture error concept. With this concept, datum-induced and machining-induced errors are transformed to equivalent fixture-induced errors at each operation. Using this approach, a sequential root cause identification can be conducted minimizing the number of measurements required, isolating firstly the faulty station. Assuming measurement and un-modeled noises to be negligible, Ding et al. [13] studied the diagnosability of an MMP through the definition of a diagnosability matrix. According to this matrix, three different types of diagnosability were defined: i) diagnosability within MMP, ii) diagnosability within station, and iii) diagnosability between stations. Zhou et al. [14] extended the diagnosability analysis of MMPs when measurement and un-modeled noises are not negligible. Besides analyzing the diagnosis capability of the MMP, other research works have also studied how to identify a specific root fault cause when it is diagnosable. For this purpose, pattern recognition techniques [16] and direct estimation methods [15] have been tested.

The definition of at which station an inspection of part/workpiece quality should be conducted and which features should be inspected is crucial for a successful identification of the root fault causes and process improvement. Djurdjanovic and Ni [17] proposed a Bayesianbased method to analyze the measurement schemes (i.e. placement of the inspection station and features to be inspected) in a MMP. Later, the same authors presented in [20] other non-Bayesian methods for analyzing different measurement schemes when only statistical characteristics of the sensor noise term $\varepsilon$ are known. Other research works such as $[18,19]$ tackle the synthesis problem to define which is the optimal placement of the inspection stations for a given MMP.

\subsubsection{Dimensional quality control}

As an extension of diagnosis methodologies, some researchers have developed an in-line process adjustment technique to reduce variability in MMPs. The basic idea is to control the product quality through in-line adjustments of certain process parameters such as the fixture locations or the cutting-tool path itself. The SoV model is applied to estimate the impact that those potential control actions will have on the quality of the final product. Active control for variation reduction requires two enablers [21]: in-line dimensional measurement sensors to measure actual part deviation, and realtime actuators such as CNC machining stations or flexible tooling [29] to act on the manufacturing process. By these enablers, dimensional quality control can be based on feed-back control or feed-forward control [21]. Feed-back control implies that the control actions (corrections) are determined using downstream measurements obtained at the end of the process or in certain 
intermediate stations. This dimensional quality control can only be used to compensate mean shifts, but not to reduce variability. On the other hand, feed-forward control uses in-line measurements to determine the current deviation of the workpiece in order to subsequently apply control actions to minimize the effect of this deviation in the final part quality. In this way, feed-forward control compensates current deviations instead of reacting to past deviations as feed-back control does [21].

The first work in the field of active control for variation reduction was conducted by Djurdjanovic and Zhu [22]. The feed-back and feed-forward control strategies for the placement of stations with dimensional adjustment capability was proposed. Innovatively addressing the dimension compensation problem, this work considers only the deterministic effects, neglecting the noise due to the linearization, unmodeled effects, process noise, and sensor imperfection. Furthermore, the concept of compensability was introduced to quantitatively evaluate the capability of variation compensation in a specific system. Izquierdo et al. [21] extended the feed-forward control strategy to include parts/process requirements and specific engineering constraints on the magnitudes of control actions, such as physical limits and inaccuracy of tooling adjustments. These works were focused on the study of feed-forward control with full control over all tooling elements. This assumption may not be realistic, since tooling adjustments through flexible fixtures or CNC machine-tools may only be assigned to selected stations in the system due to their high costs. Thus, Djurdjanovic and Ni [23] proposed a feed-forward control strategy with distributed actuation capabilities, taking into consideration the actuation accuracy and noise. However, they only select the best placement from the potential and distributed tooling adjustments, without considering the interaction of multiple tooling adjustments. Metaheuristic optimization approaches were used in $[24,25]$, where the research work in [23] was extended to deal with variation reduction considering multiple tooling adjustments. More recently, Abellan-Nebot et al. [26] proposed a methodology to implement sensor-based fixtures in MMPs analyzing at which stations the sensor-based fixture should be installed to produce the higher compensability rate, increasing the final product quality. The paper also deals with the optimal sensor distribution within the fixture to increase the compensation capability.

\subsubsection{Process-oriented tolerancing}

Process-oriented tolerancing approach is a new tolerance approach that tries to overcome the traditional limitations of the product-oriented tolerancing approach. Unlike product-oriented tolerancing, where part tolerances are optimally allocated only considering an associated manufacturing cost from very generic process planning guide-lines, process-oriented tolerancing optimally allocates tolerances of manufacturing process variables considering explicitly their associated manufacturing costs and their relationship with product quality. Basically, process-oriented tolerancing is essentially a tolerance synthesis problem where the quality specification of the final product is ensured by allocating tolerances of manufacturing process variables (such as locator tolerances) for a minimum cost. The framework of process-oriented tolerance synthesis was firstly proposed in $[28,30]$. In these research works, the tolerances of process variables in a MMP are optimally allocated by solving a non-linear constrained optimization problem defined by: cost functions, the SoV model, a process degradation model of fixture components, a tolerance accumulation model, and several constraints related to part specifications (tolerances). Chen et al. [27] expanded the work in [28] to integrate process-oriented tolerancing with maintenance planning in multi-station assembly processes. They incorporated tool fabrication cost, fixture maintenance cost and quality loss functions to optimize the tolerance allocation of manufacturing process variables and the frequency of fixture maintenance operations.

\section{Variation modeling and propagation by the PD school: The Model of the Manufactured Part}

\subsection{Fundamentals}

The PD school deals with the manufacturing variation analysis in MMPs applying some of the concepts used in analyzing the geometrical variations of mechanical assemblies due to the imperfections of their components. The main idea of the PD school is to consider the manufacturing set-up in a machining station as a mechanism, so the knowledge related to dimensional and geometrical variation analysis in mechanisms can be applied. In the literature, the study of the variation propagation in mechanisms can be conducted through different modeling approaches according to the nature of the model. The most common approaches applied are: i) kinematic models such as models based on small displacement torsors (SDTs) [31] or vector-loop based models [32,33], 
and ii) degree of freedom models such as the tolerance maps models (T-Maps) [34]. The PD school has mainly applied the SDT approach to model and propagate the surface variations from parts, fixtures and cutting-tools, deriving the so-called Model of the Manufactured Part (MoMP) [35].

The aim of the MoMP is to simulate the deviations generated in the manufacturing process considering two independent phenomena: positioning deviations and machining deviations. These deviations are accumulated over successive setups propagating the manufacturing variability. Positioning deviations are caused by fixture surface deviations and locating datum surface deviations which have been generated in previous setups. Machining deviations are caused by multiple sources of error such as geometric and kinematic errors, thermal errors, cutting force-induced errors, cuttingtool wear errors, etc. The positioning and machining deviations of part surfaces are modeled by the SDT approach assuming that the expected manufacturing variations are small and parts behave as solid rigid. By this approach, dimensional and geometrical variations of manufactured parts are obtained by propagating the deviations of the elements that take part in the manufacturing process (e.g. part-holder surfaces and workpiece surfaces), modeled as a chain of small displacement torsors.

The dimensional and geometrical deviation of each element, described by an SDT, depends on the types of surfaces and tolerances involved. An SDT of a surface is composed of the small translation and orientation deviations that define the deviation between the nominal surface and the substitute surface, which is an ideal representation of the real one. For instance, a surface with a planar geometry can only present translation variations on the $Z$-axis, and orientation variations around the $X$ - and $Y$-axes, considering the normal vector of the planar surface in $Z$ direction of its local CS. Other variations (translation in $X$ - and $Y$-axes and rotations around the $Z$-axis) keep the surface invariant and thus, these deviations are considered to be undetermined. The SDT that describes the deviation between the substitute plane $S_{i}$ and the nominal plane $N_{i}$, denoted as $\mathbf{T}_{S_{i}, N_{i}}$, is thus defined as a translation deviation vector $\mathbf{D}=U \cdot x+U \cdot y+w \cdot z$ and an orientation deviation vector $\boldsymbol{\Omega}=\alpha \cdot x+\beta \cdot y+U \cdot z$. This SDT is defined as:

$\mathbf{T}_{S_{i}, N_{i}}=\left\{\begin{array}{l}\boldsymbol{\Omega} \\ \mathbf{D}\end{array}=\left\{\begin{array}{l}\alpha \cdot x+\beta \cdot y+U \cdot z \\ U \cdot x+U \cdot y+w \cdot z\end{array}=\left\{\begin{array}{ll}\alpha & U \\ \beta & U \\ U & w\end{array}\right\}\right.\right.$ where $U$ is an undetermined component, $w$ is the translation deviation around the $Z$-axis, and $\alpha$ and $\beta$ are the orientation deviations around the $X$ - and $Y$-axes, respectively. Similar SDTs have been defined in the literature [35-37] for other types of surfaces, and some of them are shown in Table 1 . Note that the torsor components are constrained to keep the surface within the tolerance range.

In addition to the surface torsors, it is also defined the link torsor and the part torsor. The link torsor represents the link between two substitute surfaces from different parts and shows the degrees of freedom constrained by the link (joint). The part torsor represents the part's displacement within the assembly in relation with geometric errors, joints and its the nominal position of the part. For each part, a part torsor is defined and for each contact between parts, a link torsor is defined.

In assemblies, the resulting deviation of a part of an assembly can be directly computed from the summation of torsors of the assembled parts that define the position of the part analyzed. For instance, consider an assembly of two parts, $A$ and $B$. The computation of the part torsor $\mathbf{T}_{R, B}$ (SDT of part $B$ w.r.t. frame $R$ ) is evaluated for any set of joints between parts $A$ and $B$ (i.e., for any set of two interacting surfaces $\left.A_{i}, B_{j}\right)$ as follows [35]:

$$
\begin{aligned}
\mathbf{T}_{R, B} & =\mathbf{T}_{R, A}+\mathbf{T}_{A, A_{i}}+\mathbf{T}_{A_{i}, B_{j}}+\mathbf{T}_{B_{j}, B} \\
& =\mathbf{T}_{R, A}+\mathbf{T}_{A, A_{i}}+\mathbf{T}_{A_{i}, B_{j}}-\mathbf{T}_{B, B_{j}} .
\end{aligned}
$$

where $\mathbf{T}_{A, A_{i}}$ and $\mathbf{T}_{B, B_{j}}$ are the surface torsors that represent the deviation of surface $A_{i}$ and $B_{j}$ of part $A$ and $B$ respectively in the reference frame $R ; \mathbf{T}_{A_{i}, B_{j}}$ is the link torsor that represents the deviation of the link between surface $A_{i}$ and surface $B_{j}$ in the reference frame $R$; and $\mathbf{T}_{R, A}$ is the SDT of part $A$ w.r.t. frame $R$. Note that $\mathbf{T}_{R, B}$ is identical whatever the contact surface is considered, i.e., if there are $n$ interacting surfaces $A_{i}-B_{j}$ that compose the joints in the assembly of parts $A$ and $B$, thus there are $n$ equations defined as Eq. (19) that result in the same value of $\mathbf{T}_{R, B}$. In the system of $n$ linear equations, the unknown parameters are the link torsors. Obviously, different joints suppress different degrees of mobility, so for isostatic assemblies the resulting system of linear equations can be solved.

In the MoMP, the resulting variation in part quality at the end of the MMP is obtained by evaluating the chain of torsors that influence the manufacturing performance at each station, expressed all torsors in the same CS. This chain of torsors considers the positioning and the machining deviation at each station. 
Table 1 SDTs according to the type of surface and torsor constraints according to the type of tolerance.

Surface torsor

Following the research work in [35], the position and machining deviation can be evaluated as follows:

- Positioning deviation: positioning deviation, expressed as the torsor $\mathbf{T}_{F_{k}, D}$, is the deviation of the nominal design reference $(D)$ at the nominal fixture set-up $\left(F_{k}\right)$ and it is formulated as:

$$
\mathbf{T}_{F_{k}, D}=-\mathbf{T}_{D, S_{l}}+\mathbf{T}_{F_{k}, H_{k, j}}+\mathbf{T}_{H_{k, j}, S_{l}} .
$$

In this expression, $\mathbf{T}_{D, S_{l}}$ is the SDT of locating datum surfaces $\left(S_{l}\right)$ at $D$ and it is obtained from previous stations. Torsor $\mathbf{T}_{F_{k}, H_{k, j}}$ indicates the $j$ th partholder surface deviation at the $k$ station and the maximum values of the parameters of this torsor represent the part-holder precision (maximum deviations expected from part-holder surfaces). Torsor $\mathbf{T}_{H_{k, j}, S_{l}}$ represents the relative position between the locating datum surface $S_{l}$ and the $j$ th part-holder surface which depends on how both surfaces contact (joint type). The torsor $\mathbf{T}_{H_{k, j}, S_{l}}$ is also called the link torsor, and its parameters are called link parameters. Depending on the joint type of each pair of mating surfaces in the workpiece/part-holder assembly, different link torsors are defined [37]. Some of these torsors are shown in Table 2. The methodology to obtain the values of the link parameters for a generic workpiece/part-holder assembly (hyperstatic or isostatic assembly) is shown in detail in [38]. Note that Eq. (20) should be accomplished for any pair of locating datum and part-holder surfaces $S_{l}$ and $H_{k, j}$ that define the workpiece/partholder assembly.
- Machining deviation: machining deviation, expressed as the SDT $\mathbf{T}_{F_{k}, S_{i}}$ for the machining station $k$, is the deviation of the surface machining operation $\left(M_{k, o_{i}}\right.$ that generates surface $\left.S_{i}\right)$ at the nominal machinetool set-up (i.e. the $F_{k}$ ) and it is formulated as:

$\mathbf{T}_{F_{k}, S_{i}}=\mathbf{T}_{F_{k}, M_{k, o_{i}}}=\mathbf{T}_{F_{k}, M_{k, o}}+\mathbf{T}_{M_{k, o}, M_{k, o_{i}}}$,

where $\mathbf{T}_{F_{k}, M_{k, o}}$ is the SDT of the oth machining operation due to geometrical-kinematic variations and thermal distortions of the machine-tool; $\mathbf{T}_{M_{o}, M_{k, o_{i}}}$ is the SDT of the machining operation due to cuttingtool wear or cutting force-induced deviations when machining the surface $S_{i}$. It is considered that $\mathbf{T}_{F_{k}, S_{i}}$ is equal to $\mathbf{T}_{F_{k}, M_{k, o_{i}}}$, that is to say that there is an identity between the surface generated by the machining operation and the surface machined on the part. Note that the parameters of the torsors $\mathbf{T}_{F_{k}, M_{k, o}}$ and $\mathbf{T}_{M_{k, o}, M_{k, o_{i}}}$ and their constraints (maximum values) represent the machine-tools and tooling capabilities (i.e. maximum expected deviations of the cutting-tool path due to machining inaccuracies). The torsor $\mathbf{T}_{F_{k}, M_{k, o_{i}}}$ will be defined according to the type of the surface geometry generated and the capability of the manufacturing process. For instance, for a face milling operation that generates a planar surface parallel to the machine-tool table, the following torsor will be defined:

$\mathbf{T}_{F_{k}, M_{k, o_{i}}}=\left\{\begin{array}{ll}\alpha_{M_{i}}^{k} & U \\ \beta_{M_{i}}^{k} & U \\ U & w_{M_{i}}^{k}\end{array}\right\}$,

where $w_{M_{i}}^{k}, \alpha_{M_{i}}^{k}$ and $\beta_{M_{i}}^{k}$ are the machining deviations (translation and orientation deviations). 
Table 2 SDTs (link torsors) according to the joint type of mating surfaces.

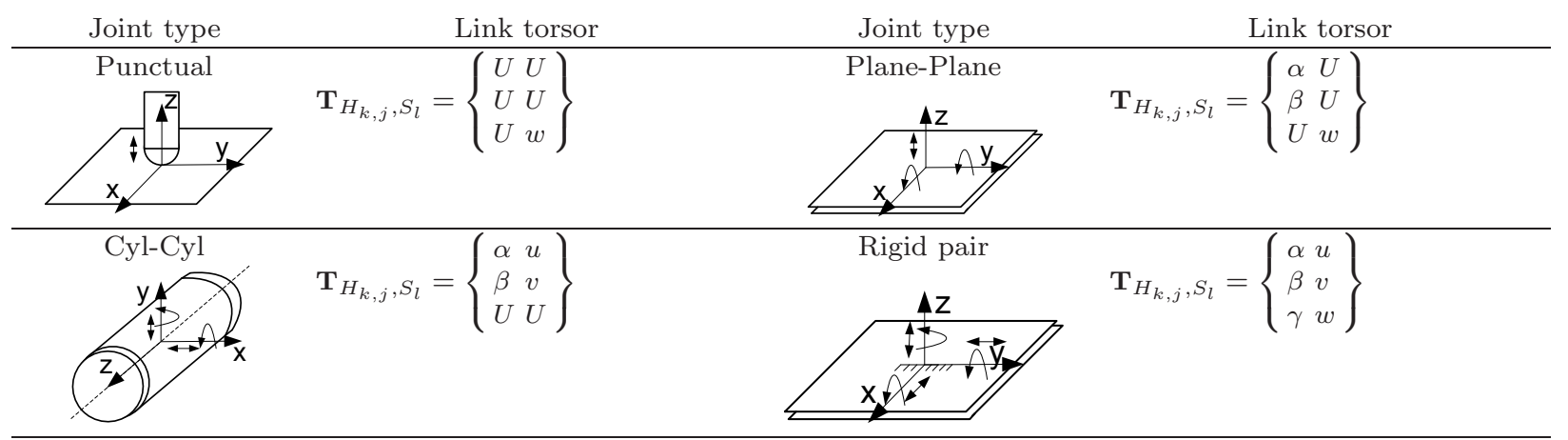

According to the positioning deviation and the machining deviation, the deviation of the actual part surface at the D CS in a single set-up, defined by the SDT $\mathbf{T}_{D, S_{i}}$, can be evaluated as follows:

$\mathbf{T}_{D, S_{i}}=-\mathbf{T}_{F_{k}, D}+\mathbf{T}_{F_{k}, S_{i}}$.

Note that the resulting torsors $\mathbf{T}_{D, S_{i}}, \mathbf{T}_{D, S_{i+1}}, \ldots$ related to the $i$ th, $(i+1)$ th, ... manufacturing features at one station may be used as inputs in subsequent stations in case that these features are used as locating datums. Thus, the deviation of machined surfaces are propagated in the subsequent stations through the SDT $\mathbf{T}_{F_{k}, D}$. As shown in Figure 4, the station-by-station evaluation of all torsors defines the MoMP.

The derivation of the MoMP was firstly proposed by Villeneuve et al. [35] for milling processes, considering both isostatic and hyperstatic fixtures, the later with a specific hierarchy of positioning surfaces (primary, secondary and tertiary positioning surfaces). Vignat and Villeneuve [39] extended the formulation of MoMP for modeling manufacturing variation in turning processes considering negligible the vibration effects and the rotation defect of the lathe. The generic resolution of the positioning problem between workpiece and part-holder was studied by Villeneuve and Vignat [38] providing a straightforward methodology to obtain the values of the link parameters. More recently, Nejad et al. [40] proposed the combination of the MoMP and the unified Jacobian-torsor model developed by Ghie et al. [41] for modeling mechanical assemblies. They used the interval arithmetic to associate bounds on the components of small displacement torsors in order to determine the lower an upper limit between which the actual surfaces must lie.
3.2 Virtual part quality inspection and verification

The formulation of the MoMP ends with the inclusion of the virtual inspection of the part using a virtual gauge. The virtual gauge is a perfect gauge made up of positioning surfaces and tolerance zone surfaces. The gauge and the resulting part from the MoMP are assembled and, similar to the assembly process of the workpiece/part-holder in the machining setup, a positioning deviation can be defined when the virtual inspection is conducted (see Figure 4). For the virtual gauge, the gauge CS $G$ and gauge surfaces are defined, the $p$ th gauge surface being defined as $G_{p}$. The gauge positioning deviation is defined as:

$\mathbf{T}_{D, G}=-\mathbf{T}_{G, G_{p}}+\mathbf{T}_{D, S_{m}}+\mathbf{T}_{S_{m}, G_{p}}$,

where torsor $\mathbf{T}_{G, G_{p}}$ indicates the deviation of the positioning surface $p$ of the gauge and the maximum values of the parameters of this torsor represent the gauge precision (if we assume the inaccuracy of the gauge to be negligible, $\left.\mathbf{T}_{G, G_{p}}=\left\{\begin{array}{ll}\mathbf{0}_{3 \times 1} & \mathbf{0}_{3 \times 1}\end{array}\right\}\right)$; torsor $\mathbf{T}_{D, S_{m}}$ represents the deviation of the measurement datum surface $S_{m}$; and the link torsor $\mathbf{T}_{S_{m}, G_{p}}$ represents the relative position between $S_{m}$ and the positioning gauge surface $G_{p}$, which depends on the part/gauge assembly condition (joint type).

After assembling the gauge and the final part, the functional tolerance compliance is verified by measuring the signed distance between the virtual gauge and the inspected surface $S_{q}$. This distance is evaluated at the boundary points of the toleranced surface projected along the inspection direction, obtaining the distance $\operatorname{Gap}_{r}$ for each $r$ th boundary point. To calculate the $G a p_{r}$ distance, first, the deviation between the inspected surface and its tolerance zone (defined as $T Z_{q}$ ) should be calculated. This deviation is expressed with the SDT $\mathbf{T}_{T Z_{q}, S_{q}}$, and it is evaluated following the Eq. (25) (see Ref. [42]):

$\mathbf{T}_{T Z_{q}, S_{q}}=-\mathbf{T}_{D, G}+\mathbf{T}_{D, S_{q}}-\mathbf{T}_{G, T Z_{q}}$, 


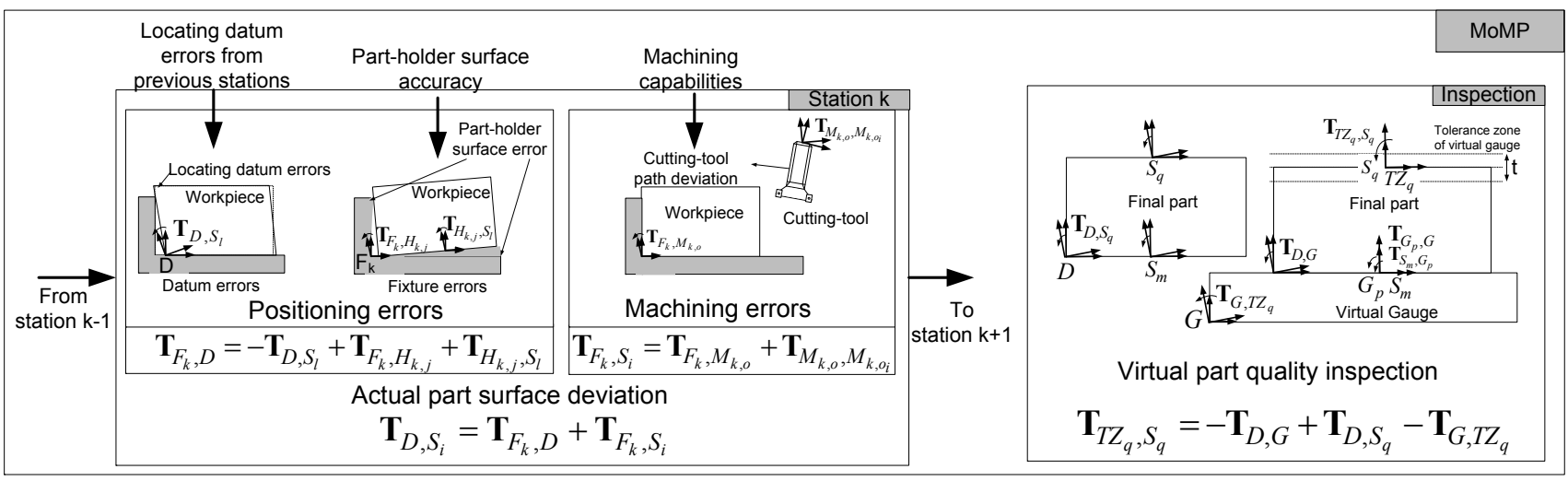

Fig. 4 Model of Manufactured Part throughout the MMP.

where $\mathbf{T}_{D, S_{q}}$ represents the deviation of the surface to be inspected $S_{q}$ and $\mathbf{T}_{G, T Z_{q}}$ is the deviation of the tolerance zone of the inspected surface w.r.t. the gauge CS, which is assumed to be $\left\{\begin{array}{ll}\mathbf{0}_{3 \times 1} & \mathbf{0}_{3 \times 1}\end{array}\right\}$ if gauge errors are negligible. Considering the $\mathrm{SDT} \mathbf{T}_{T Z_{q}, S_{q}}$ as follows:

$\mathbf{T}_{T Z_{q}, S_{q}}=\left\{\begin{array}{l}\boldsymbol{\Omega}_{T Z_{q}, S_{q}} \\ \mathbf{D}_{T Z_{q}, S_{q}}\end{array}\right\}$,

the SDT that defines the deviation of the $r$ th boundary point of the toleranced surface $S_{q}$ is expressed as:

$\mathbf{T}_{T Z_{q}, P_{r}}=\left\{\begin{array}{l}\boldsymbol{\Omega}_{T Z_{q}, P_{r}} \\ \mathbf{D}_{T Z_{q}, P_{r}}\end{array}\right\}=\left\{\begin{array}{l}\boldsymbol{\Omega}_{T Z_{q}, S_{q}} \\ \mathbf{D}_{T Z_{q}, S_{q}}+\boldsymbol{\Omega}_{T Z_{q}, S_{q}} \times \mathbf{t}_{P_{r}}^{S_{q}}\end{array}\right\}$,

where $\mathbf{t}_{P_{r}}^{S_{q}}$ is the translation vector from $S_{q}$ to $P_{r}$, and $\times$ is the cross product operator. By knowing the SDT $\mathbf{T}_{T Z_{q}, P_{r}}$, the deviation of the $r$ th point of the toleranced surface along the direction of part verification, defined by the vector $\mathbf{n}=\left[n_{x}, n_{y}, n_{z}\right]^{T}$, is evaluated by the expression:

$\left[\mathbf{T}_{T Z_{q}, P_{r}}\right]_{\mathbf{n}}=\mathbf{n} \cdot \mathbf{D}_{T Z_{q}, P_{r}}$

Analyzing the distance between the point deviation and the tolerance zone, the gap distance defined as $\mathrm{Gap}_{r}$ is formulated as:

$\operatorname{Gap}_{r}=\min \left(\tau+\left[\mathbf{T}_{T Z_{q}, P_{r}}\right]_{\mathbf{n}}, \tau-\left[\mathbf{T}_{T Z_{q}, P_{r}}\right]_{\mathbf{n}}\right)$

where $\tau$ is the maximum deviation of the $r$ th boundary point according to the tolerance value. The $r$ th boundary point of the inspected surface will be within the tolerance zone if $\mathrm{Gap}_{r}$ remains positive or null.

In a similar way to the virtual verification by the SoV model, the virtual measurement and verification by the MoMP can be conducted following two main approaches: the worst-case approach and the statistical approach. For each approach, the resulting estimation is derived as follows:
- Worst case approach:

Villeneuve and Vignat [43] reported that for a worstcase analysis, the tolerance compliance is conducted by solving an optimization problem in which the minimum gap distance from Eq. (29) at all boundary points of the toleranced surface is evaluated. This optimization problem is defined as:

Gap $_{w c}=\min _{D M, D H, L H P}^{C M, C H, C G P}\left(\max _{L G P}^{C G P}\left(\operatorname{Gap}_{\min }\right)\right)$,

where

$\operatorname{Gap}_{\min }=\min \left(\operatorname{Gap}_{r}\right), \quad \forall r \in$ boundary points.

In Eq. (30), the term Gapmin is the minimum distance between the virtual gauge and the toleranced surface inspected after measuring the distance at all boundary points. The expression $\max _{L G P}^{C G P}\left(\right.$ Gap $\left._{\text {min }}\right)$ defines the inspection process, where the gauge is assembled with the part according to the standard ISO or ASME tolerance specifications shown in the design drawings. The resulting assembly depends on how the part is inspected (defined by the link parameters denoted as LGP, which are the parameters of the torsor $\mathbf{T}_{S_{m}, G_{p}}$ ) and the positioning limits defined by the constraints of the positioning algorithm (denoted as CGP), as explained in [38]. Within the limits of these displacements, the most favorable position for the virtual gauge relative to the part can be found by maximizing the Gap min $_{\text {value. In Eq. }}$ (30), material condition modifiers or incomplete datum frames can be considered in the tolerance verification, since they are related to the link parameters LGP of the gauge/part assembly and the positioning constraints CGP. The term $\min _{D M, D H, L H P}^{C M, C H, C G P}(\cdot)$ is the search expression of the worst-case combination of the manufacturing defects DM, DH, LHP (machining, part-holder, and workpiece-fixture assembly deviations, respectively) within the estimated 
range of variations expressed by the constraints CM, $\mathrm{CH}$, CHP (machining, part-holder, and workpiecefixture assembly constraints, respectively, which are related to machine-tool and fixture capabilities and workpiece-fixture configurations). According to this worst-case analysis, a process plan will be considered able to satisfy the functional tolerance if $G_{a p}$ remains positive or null, which means that the deviation of the inspected surface is within the tolerance zone defined in the part drawing.

- Statistical approach: As reported above, the worstcase search is defined in Eq. (30) by the term $\min _{D M, D H, L H P}^{C M, C H, C G P}(\cdot)$. For a statistical analysis, instead of conducting a search for the worst-case combination, a large number of simulations are conducted in which the sources of variation DM, DH, LHP (machining, part-holder, and workpiece-fixture assembly deviations, respectively) are simulated following a specific probability distribution function. For each simulation, the Gap min is evaluated. After running thousands of simulations, the resulting probability distribution of the variable $G_{a p_{\text {min }}}$ defines whether, statistically, the parts comply with the functional tolerances [44].

\subsection{Main applications}

Basically, two main applications of the MoMP are distinguished: tolerance analysis [40, 42, 45-48] and tolerance synthesis $[43,49,50]$.

\subsubsection{Tolerance analysis}

The purpose of a tolerance analysis is to verify whether the design tolerance requirements can be met for a given process plan with specified manufacturing deviations. In tolerance analysis, the cumulative effect of individual variations with respect to the specified functional tolerance in all machining operations is studied in order to check a products functionality compared with its design requirements. This is also referred to as error propagation, error stack-up, and tolerance stack-up in a MMP.

In this field, Ayadi et al. [45] applied the MoMP for MMPs based on 3-2-1 generic fixtures. The proposed an ascendant transfer method to simplify the resolution of the virtual inspection equation (e.g. the worst case analysis given by Eq. (30)) and make possible to run the tolerance analysis more straightforward. Louati et al. [46] applied the MoMP to quantify the part quality variation due to different setups in order to select the best setting solution. Tichadou et al. [47] compared the use of the MoMP and an integrated CAD/CAM system for tolerance analysis in MMPs remarking the acceptable accuracy of the MoMP according to common requirements in mechanics, and the difficulty of translating the GD\&T specifications to be applied within the MoMP. Nejad et al. [42] proposed a detailed mathematical formulation of tolerance analysis based on searching the worst case using two different optimization methods such as genetic algorithms and sequential quadratic programming. To simplify the resolution of Eq. (30), the optimization problem was broken down into two subproblems: the worst possible part produced according to the MMPs, and the optimal inspection of one individual part. The resolution of the first subproblem is considered the input for the second subproblem. The tolerance analysis presented also considered different strategies to estimated the constraints related to the deviation torsor parameters of fixture and machine tool's capabilities. Nejad et al. [40] studied the tolerance analysis problem using a combined approach of the MoMP and the JacobianTorsor model. This work applied the interval arithmetic so all torsors were expressed by interval ranges. The worst-case analysis is obtained studying the error stack-up on the functional elements expressed in interval ranges, and makes the resolution quite rapid compared with previous methods. However, the approach is nonetheless limited by the fact that it considers the torsor parameters independently, so they can reach their extreme values simultaneously which is not realistic. A detail comparison of both solution techniques presented in [40,42] was reported in [48], considering both worst-case and statistical analysis. The comparison showed that the interval approach performance is faster and accurate when using the simple quality constraints whereas the first approaches were time consuming but allows the realistic quality constraints. Additionally, the comparison was also conducted applying different strategies to simulate the torsors related to the performance of fixtures and machine-tools (constraints parameters that define the machine-tool capability and fixture accuracy). These strategies were previously studied in other research works $[51,52]$.

\subsubsection{Tolerance synthesis}

From the functional requirements of a part, the manufacturing requirements at each setup can be derived using the MoMP. In fact, from the functional inequalities system the corresponding manufacturing inequalities are determined. These inequalities limit the defect allowed for each setup to produce a final part con- 
form with the functional tolerance. The general tolerance synthesis problem is presented in [43]. Anselmetti and Louati [49] described in detail the tolerance synthesis considering the ISO standard. A simple algorithm is used to directly provide a complete set of manufacturing specifications in compliance with ISO standards, with the orientation and location specifications and datum reference frame. The method is applied for each functional requirements, deriving for each case the corresponding manufacturing specifications. Similarly, Vignat and Villeuve [50] studied the derivation of ISO manufacturing tolerances for each station. By their method, it can be determined if the proposed set of manufacturing tolerances is complete and if there are unnecessary manufacturing specifications whose can be eliminated from the optimization problem.

\section{Modeling examples}

\subsection{Modeling example: SoV model}

For illustrative purposes of the SoV model derivation, consider the part design and its associated raw material shown in Fig. 5, and the 2-station machining process used in the manufacturing process shown in Fig. 6. In order to evaluate the final part variability due to both fixture- and machining-induced deviations, the SoV methodology was applied. The methodology to derive the SoV model can be summarized in the following steps:

- Step 1: Define the CSs of fixtures and part surfaces. For the case study, Tables 3 and 4 are defined.

- Step 2: Define the coordinates of fixture locators w.r.t. $F_{k}$, as it is also shown in Table 4 .

- Step 3: For the first station, define the deviation of the raw part surfaces from nominal values. Without loss of generality, we assume nominal values of raw material surfaces (initial surface deviations are negligible), so $\mathbf{x}_{0}=\mathbf{0}_{42 \times 1}$.

- Step 4: For each $k$ station, derive the vector $\mathbf{u}_{k}$. For the case study, the vector $\mathbf{u}_{k}$ is defined as $\left[\left(\mathbf{u}_{k}^{f}\right)^{T}\right.$, $\left.\left(\mathbf{u}_{k}^{m}\right)^{T}\right]^{T}$, where $\mathbf{u}_{k}^{f}$ are the locator deviations at station $k$ defined by $\left[\Delta l_{1 y}^{k}, \Delta l_{2 y}^{k}, \Delta l_{3 x}^{k}\right]^{T}$, and $\Delta l_{j *}^{k}$ refers to the deviation of the $j$ th locator in the $*$ direction; and $\mathbf{u}_{k}^{m}$ are the machining deviations when machining surface $i$, defined by $\left[u_{M_{i}}^{k}, v_{M_{i}}^{k}, 0,0,0, \gamma_{M_{i}}^{k}\right]^{T}$, where $u_{M_{i}}^{k}, v_{M_{i}}^{k}$ and $\gamma_{M_{i}}^{k}$ refer to the translation deviation of the cutting-tool path along $X$ - and $Y$ axis, and orientation deviations around $Z$-axis, respectively.

- Step 5: For each $k$ station, derive the matrices $\mathbf{A}_{k}$ and $\mathbf{B}_{k}$ as shown in [4].
Table 3 Nominal location and orientation of each local feature CS. Dimensions in $\mathrm{mm}$ and $\mathrm{rad}$.

\begin{tabular}{cccccc}
$\mathrm{CS}$ & $\left(\boldsymbol{\varphi}_{S_{i}}^{D}\right)^{T}$ & $\left(\mathbf{t}_{S_{i}}^{D}\right)^{T}$ & $\mathrm{CS}$ & $\left(\boldsymbol{\varphi}_{S_{i}}^{D}\right)^{T}$ & $\left(\mathbf{t}_{S_{i}}^{D}\right)^{T}$ \\
\hline$S_{1}$ & {$[0,0,0]$} & {$[37.5,50,0]$} & & & \\
$S_{2}$ & {$[0,0, \pi / 2]$} & {$[75,25,0]$} & $S_{5}$ & {$[0,0,0]$} & {$[37.5,45,0]$} \\
$S_{3}$ & {$[0,0, \pi]$} & {$[37.5,0,0]$} & $S_{6}$ & {$[0,0, \pi]$} & {$[70,5,0]$} \\
$S_{4}$ & {$[0,0,-\pi / 2]$} & {$[0,25,0]$} & $S_{7}$ & {$[0,0, \pi / 2]$} & {$[65,2.5,0]$} \\
\hline
\end{tabular}

Table 4 Nominal location and orientation of Fixture CS $\left(F_{k}\right)$ at each station. Position of locators is also shown. Dimensions in $\mathrm{mm}$ and $\mathrm{rad}$.

\begin{tabular}{cccc}
$F_{k}$ & $\left(\boldsymbol{\varphi}_{F_{k}}^{D}\right)^{T}$ & $\left(\mathbf{t}_{F_{k}}^{D}\right)^{T}$ & Locators w.r.t. $F_{k}$ \\
\hline$F_{1}$ & {$[0,0,0]$} & {$[0,0,0]$} & $l_{1 x}=25, l_{1 y}=0, l_{2 x}=50$, \\
& & & $l_{2 y}=0, l_{3 x}=0, l_{3 y}=22.5$ \\
$F_{2}$ & {$[0,0, \pi]$} & {$[75,45,0]$} & $l_{1 x}=25, l_{1 y}=0, l_{2 x}=50$, \\
& & & $l_{2 y}=0, l_{3 x}=0, l_{3 y}=22.5$ \\
\hline
\end{tabular}

- Step 6: Derive the matrix $\mathbf{C}_{K}$ where $K$ is the inspection station, as shown in [4].

- Step 7: For each KPCs, evaluate the deviation of the boundary points of each toleranced surface w.r.t. the measurement datum by Eqs. (10-12).

\subsubsection{Symbolic resolution}

Applying the SoV model for the 2D case study, the resulting deviation of each KPC due to fixture- and machining-induced deviations is defined as follows:

- $K P C_{1}$ : the deviation of the $K P C_{1}$ is defined as

$$
K P C_{1}=\max \left(\left|\left[\mathbf{y}_{3, P_{6 A}}\right]_{\mathbf{y}}\right|,\left|\left[\mathbf{y}_{3, P_{6 B}}\right]_{\mathbf{y}}\right|\right),
$$

where these deviations are related to fixture and machining deviations as:

$$
\begin{aligned}
{\left[\mathbf{y}_{3, P_{6 A}}\right]_{\mathbf{y}}=} & -27.5 \gamma_{M_{5}}^{1}+5 \gamma_{M_{6}}^{2}-1.6 \Delta l_{1 y}^{2}+0.6 \Delta l_{2 y}^{2} \\
& -0.6 \Delta l_{1 y}^{1}+1.6 \Delta l_{2 y}^{1}-v_{M_{5}}^{1}+v_{M_{6}}^{2},(33) \\
{\left[\mathbf{y}_{3, P_{6 B}}\right]_{\mathbf{y}}=} & -37.5 \gamma_{M_{5}}^{1}-5 \gamma_{M_{6}}^{2}-2 \Delta l_{1 y}^{2}+2 \Delta l_{2 y}^{1} \\
& +\Delta l_{2 y}^{2}-\Delta l_{1 y}^{1}-v_{M_{5}}^{1}+v_{M_{6}}^{2} .
\end{aligned}
$$

Note that most of the deviations in the first station are propagated downstream, affecting the $K P C_{1}$. However, note that locator deviations $\Delta l_{3 x}^{1}$ and $\Delta l_{3 x}^{2}$ and machining deviations along $X$ direction $\left(u_{M_{5}}^{1}\right.$ and $u_{M_{6}}^{2}$ ) do not influence on $K P C_{1}$.

- $K P C_{2}$ : the deviation of the $K P C_{2}$ is defined as

$K P C_{2}=\max \left(\left|\left[\mathbf{y}_{4, P_{7 A}}\right]_{\mathbf{y}}\right|,\left|\left[\mathbf{y}_{4, P_{7 B}}\right]_{\mathbf{y}}\right|\right)$, 


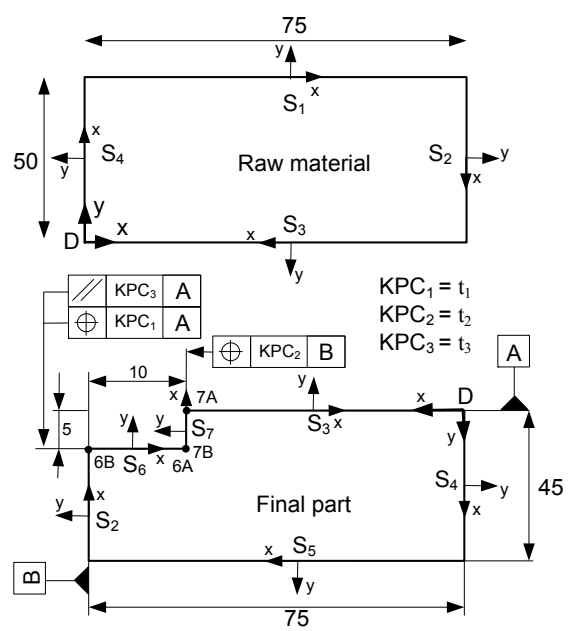

Fig. 5 2D case study. Raw material and part design. Dimensions in $\mathrm{mm}$.

where

$$
\begin{aligned}
{\left[\mathbf{y}_{4, P_{7 A}}\right]_{\mathbf{y}}=} & +22.5 \gamma_{M_{5}}^{1}+2.5 \gamma_{M_{7}}^{2}+0.9 \Delta l_{1 y}^{1}-0.9 \Delta l_{2 y}^{1} \\
& -0.9 \Delta l_{2 y}^{2}+0.9 \Delta l_{1 y}^{2}+\Delta l_{3 x}^{2}-u_{M_{7}}^{2}, \quad(36) \\
{\left[\mathbf{y}_{4, P_{7 B}}\right]_{\mathbf{y}}=} & +17.5 \gamma_{M_{5}}^{1}-2.5 \gamma_{M_{7}}^{2}+0.7 \Delta l_{1 y}^{1}-0.7 \Delta l_{2 y}^{1} \\
& -0.7 \Delta l_{2 y}^{2}+0.7 \Delta l_{1 y}^{2}+\Delta l_{3 x}^{2}-u_{M_{7}}^{2} .
\end{aligned}
$$

In this case, note that the locator deviations $\Delta l_{3 x}^{1}$ do not influence on this $\mathrm{KPC}$, however the same locator deviation in station $2\left(\Delta l_{3 x}^{2}\right)$ does influence. Furthermore, only machining deviations when milling surface $S_{7}$ influence on the KPC (except the deviation $v_{M_{7}}^{2}$ ), and the machining deviations when milling surface $S_{6}$ and $S_{5}$ do not influence at all.

$-K P C_{3}$ : the deviation of $K P C_{3}$ is defined as

$K P C_{3}=\left|\left[\mathbf{y}_{3, P_{6 A}}\right]_{\mathbf{y}}-\left[\mathbf{y}_{3, P_{6 B}}\right]_{\mathbf{y}}\right|$,

where $\left[\mathbf{y}_{3, P_{6 A}}\right]_{\mathbf{y}}$ and $\left[\mathbf{y}_{3, P_{6 B}}\right]_{\mathbf{y}}$ are defined by Eqs. (33) and (34) respectively. By substituting, the deviation of $K P C_{3}$ is defined as $\mid 10 \gamma_{M_{5}}^{1}+10 \gamma_{M_{6}}^{2}+$ $0.4 \Delta l_{1 y}^{1}-0.4 \Delta l_{2 y}^{1}-0.4 \Delta l_{2 y}^{2}+0.4 \Delta l_{1 y}^{2} \mid$. Note that as this $\mathrm{KPC}$ is a parallelism relationship, locator deviations $\Delta l_{3 x}^{1}$ and $\Delta l_{3 x}^{2}$, and translational machining deviations at any station do not influence.

\subsubsection{Numerical resolution}

The case study is numerically solved analyzing the worstcase and the statistical approach. The expected variability range for each manufacturing process variable is

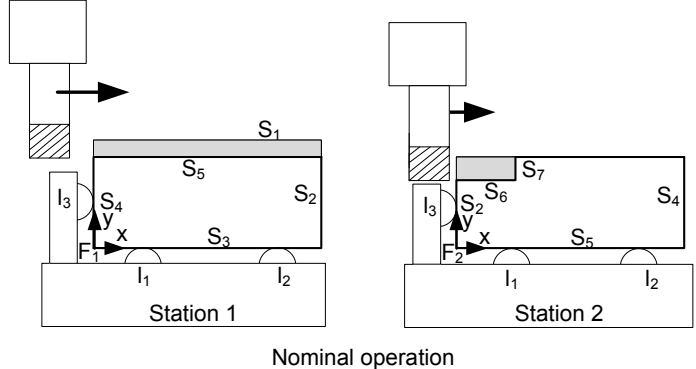

Fig. 6 Multi-station machining process to manufacture the $2 \mathrm{D}$ case study.

Table 5 Ranges of locators and machining deviations for the SoV case study. Dimensional deviations in -mm-, angular deviations in -rad-.

\begin{tabular}{llllll}
\multicolumn{2}{l}{ Station 1} \\
\hline$\Delta l_{1 y}^{1}$ & \pm 0.02 & $\Delta l_{2 y}^{1}$ & \pm 0.02 & $\Delta l_{3 x}^{1}$ & \pm 0.02 \\
$u_{M_{5}}^{1}$ & \pm 0.01 & $v_{M_{5}}^{1}$ & \pm 0.01 & $\gamma_{M_{5}}^{1}$ & \pm 0.001 \\
\hline
\end{tabular}

\begin{tabular}{llllll}
\multicolumn{2}{l}{ Station 2} & & & & \\
\hline$\Delta l_{1 y}^{2}$ & \pm 0.02 & $\Delta l_{2 y}^{2}$ & \pm 0.02 & $\Delta l_{3 x}^{2}$ & \pm 0.02 \\
$u_{M_{6}}^{2}$ & \pm 0.01 & $v_{M_{6}}^{2}$ & \pm 0.01 & $\gamma_{M_{6}}^{2}$ & \pm 0.001 \\
$u_{M_{7}}^{2}$ & \pm 0.01 & $v_{M_{7}}^{2}$ & \pm 0.01 & $\gamma_{M_{7}}^{2}$ & \pm 0.001 \\
\hline
\end{tabular}

Table 6 Numerical resolution according to the worst-case (WC) and the statistical (ST) analysis for the SoV case study. Dimensions in $-m m-$.

\begin{tabular}{l|ccc} 
& $K P C_{1}$ & $K P C_{2}$ & $K P C_{3}$ \\
\hline WC (Pro/E) & \pm 0.183 & \pm 0.127 & \pm 0.051 \\
WC & \pm 0.182 & \pm 0.127 & \pm 0.052 \\
ST (6 $\sigma$ interval) & \pm 0.075 & \pm 0.048 & \pm 0.021 \\
\hline
\end{tabular}

shown in Table 5 and all manufacturing process variables are assumed to be independent to each other. For the statistical analysis the manufacturing process variables are assumed to be normally distributed and the ranges shown in Table 5 cover the $6 \sigma$ interval. In order to validate the model, the worst-case analysis was also conducted using Pro/Engineer Wildfire 5.0. Using this software, the assembly of the workpiece and the fixture at each machining station was generated, and a material removal operation to simulate the machining operation was also defined. As a result, the final machined part was obtained as a function of the previous assemblies and operations. The worst-case analysis was then conducted by analyzing the resulting part at the extreme values of the manufacturing sources of variation and measuring all KPCs. Table 6 shows the KPC variations according to the type of analysis conducted. 


\subsection{Modeling example: MoMP model}

For illustrative purposes, the same $2 \mathrm{D}$ case study shown in Fig. 5 is used to describe the use of the MoMP. Unlike the previous example, the current 2-station manufacturing process applies fixture surfaces instead of fixture locators as it is shown in Fig. 7, since this modeling approach lets model surface-based fixtures. The MoMP is built applying the methodology composed of the following steps:

- Step 1: Define the CSs for parts, fixtures, gauge, part surfaces, fixture surfaces and gauge surfaces. For this example, Tables 3 (the same as in the SoV example), 7 and 8 apply.

- Step 2: Define the coordinates of fixture surfaces at the fixture CS and the coordinates of gauge surfaces at the $G$ CS. For the case study, Tables 7 and 8 also show this information.

- Step 3: For the first station, define the deviation of the raw part surfaces from nominal values. Without loss of generality, we assume nominal values of raw material surfaces, so $\left\{\mathbf{T}_{D, S_{l}}\right\}_{D}=\left\{\begin{array}{ll}\mathbf{0}_{3 \times 1} & \mathbf{0}_{3 \times 1}\end{array}\right\}$ for $l=1,2,3$ and 4 . A torsor $\left\{\mathbf{T}_{\bullet}, \bullet\right\}_{D}$ refers to a torsor expressed in the frame $D$. Note that torsors should be expressed in the same frame to be summed.

- Step 4: For the next $k$ th machining station (starting from station 1), derive the following torsors:

$-\left\{\mathbf{T}_{F_{k}, H_{k, j}}\right\}_{D}$, according to fixture accuracy.

- $\left\{\mathbf{T}_{H_{k, j}, S_{l}}\right\}_{D}$, according to each workpiece/partholder joint.

- $\left\{\mathbf{T}_{D, S_{l}}\right\}_{D}$, according to surface deviations from previous stations.

- Step 5: Derive the SDT $\left\{\mathbf{T}_{F_{k}, D}\right\}_{D}$ by Eq. (20) following the methodology shown in [38], taking into account the datum hierarchy (primary and secondary datums).

- Step 6: Derive the SDT $\left\{\mathbf{T}_{F_{k}, M_{k, o_{i}}}\right\}_{F_{k}}$ by Eq. (21) according to the machine-tool precision.

- Step 7: Derive the torsor $\left\{\mathbf{T}_{D, S_{i}}\right\}_{D}$ by Eq. (23).

- Step 8: Repeat the steps 4-7 for all machining stations. Note that some SDTs in one station depend on other SDTs from previous stations, so the resolution of the problem should be station by station starting from upstream stations and propagating the results in downstream stations.

- Step 9: To measure the deviation of the KPCs by a virtual gauge, derive the following torsors:

- $\left\{\mathbf{T}_{G, G_{p}}\right\}_{D}$, according to gauge accuracy. Without loss of generality, we can assume negligible this deviation, so $\left\{\mathbf{T}_{G, G_{p}}\right\}_{D}=\left\{\begin{array}{ll}\mathbf{0}_{3 \times 1} & \mathbf{0}_{3 \times 1}\end{array}\right\}$.

$-\left\{\mathbf{T}_{S_{m}, G_{p}}\right\}_{D}$, according to each part/gauge-surface joint.
Table 7 Nominal location and orientation of $F_{k}$ and $H_{j}$. Dimensions in $\mathrm{mm}$ and $\mathrm{rad}$.

\begin{tabular}{cccccc}
$F_{k}$ & $\left(\varphi_{F_{k}}^{D}\right)^{T}$ & $\left(\mathbf{t}_{F_{k}}^{D}\right)^{T}$ & $H_{j}$ & $\left(\boldsymbol{\varphi}_{H_{j}}^{F_{k}}\right)^{T}$ & $\left(\mathbf{t}_{H_{j}}^{F_{k}}\right)^{T}$ \\
\hline$F_{1}$ & {$[0,0,0]$} & {$[0,0,0]$} & $H_{1}$ & {$[0,0,0]$} & {$[37.5,0,0]$} \\
& & & $H_{2}$ & {$[0,0, \pi / 2]$} & {$[0,10,0]$} \\
$F_{2}$ & {$[0,0, \pi]$} & {$[75,45,0]$} & $H_{3}$ & {$[0,0,0]$} & {$[37.5,0,0]$} \\
& & & $H_{4}$ & {$[0,0, \pi / 2]$} & {$[0,10,0]$} \\
\hline
\end{tabular}

Table 8 Nominal location and orientation of $G$ and gauge surfaces $G_{p}$. Dimensions in $m m$ and $r a d$.

\begin{tabular}{cccccc}
$\mathrm{KPC}$ & $\left(\boldsymbol{\varphi}_{G}^{D}\right)^{T}$ & $\left(\mathbf{t}_{G}^{D}\right)^{T}$ & $G_{p}$ & $\left(\boldsymbol{\varphi}_{G_{p}}^{G}\right)^{T}$ & $\left(\mathbf{t}_{G_{p}}^{G}\right)^{T}$ \\
\hline$K P C_{1}$ & {$[0,0,0]$} & {$[0,0,0]$} & $G_{1}$ & {$[0,0,0]$} & {$[37.5,0,0]$} \\
$K P C_{2}$ & {$[0,0, \pi / 2]$} & {$[-45,75,0]$} & $G_{2}$ & {$[0,0,0]$} & {$[-25,0,0]$} \\
$K P C_{3}$ & {$[0,0,0]$} & {$[0,0,0]$} & $G_{1}$ & {$[0,0,0]$} & {$[37.5,0,0]$} \\
\hline
\end{tabular}

$-\left\{\mathbf{T}_{D, S_{m}}\right\}_{D}$, according to surface deviations from previous stations.

By these SDTs and applying Eq. (24), the positioning deviation torsor for the gauge/fixture assembly $\left(\left\{\mathbf{T}_{D, G}\right\}_{D}\right)$ can be evaluated. Then, the deviation torsor between the actual toleranced surface and its tolerance zone is evaluated by Eq. (25). Finally, the deviation of each $r$ th boundary point of the toleranced surface from its nominal value is evaluated by Eq. (29).

\subsubsection{Symbolic resolution}

Following this methodology, the final part variability of each KPC due to fixture- and machining-induced deviations can be obtained. For the 2D case study shown in Fig. 7, the resulting torsor of positioning deviation in each station depends on how the part contacts on the secondary part-holder surface. In fact, there are different possible workpiece/fixture configurations since the workpiece can be located in the $\mathrm{X}$ direction by contacting on point $\mathrm{B}$ or $\mathrm{A}$ in the station 1 , and on point I or E in station 2, assuming no form error exists. At which point the workpiece contacts on the secondary part-holder surface depends on the deviations of partholder and workpiece surfaces. For the 2D case study, the contact at point $\mathrm{B}$ in the first station and at point I in the second station occurs if, applying the resolution of the generic positioning problem shown in [38], Eqs. (39) and (40) apply for station 1 and 2 respectively.

$\gamma_{H_{2}, S_{4}}^{1}=\left(\gamma_{H_{1}}^{1}-\gamma_{H_{2}}^{1}\right)>0$,

$\gamma_{H_{4}, S_{2}}^{2}=\left(\gamma_{H_{3}}^{2}-\gamma_{M_{5}}^{1}+\gamma_{H_{1}}^{1}-\gamma_{H_{4}}^{2}\right)>0$.

If Eqs. (39) and (40) hold, the deviation of the joint workpiece/fixture at the secondary locating datum in $X$ 
direction (expressed in the fixture CS) at each station is defined as:

$v_{H_{2}, S_{4}}^{1}=-p_{B}^{F_{1}} \cdot\left(\gamma_{H_{1}}^{1}-\gamma_{H_{2}}^{1}\right)$,

$v_{H_{4}, S_{2}}^{2}=-p_{I}^{F_{2}} \cdot\left(\gamma_{H_{3}}^{2}-\gamma_{M_{5}}^{1}+\gamma_{H_{1}}^{1}-\gamma_{H_{4}}^{2}\right)$.

For these workpiece / fixture configurations at station 1 and 2, the resulting final part variability is defined as follows:

- $K P C_{1}$ : the deviation of $K P C_{1}$ is defined as

$K P C_{1}=\max \left(\left|\left[\mathbf{T}_{T Z_{1}, P_{6 A}}\right]_{\mathbf{y}}\right|,\left|\left[\mathbf{T}_{T Z_{1}, P_{6 B}}\right]_{\mathbf{y}}\right|\right)$,

where:

$$
\begin{aligned}
{\left[\mathbf{T}_{T Z_{1}, P_{6 A}}\right]_{\mathbf{y}}=} & -v_{H_{1}}^{1}+v_{M_{5}}^{1}+v_{H_{3}}^{2}-v_{M_{6}}^{2}-5 \gamma_{M_{6}}^{2} \\
& +27.5 \cdot\left(\gamma_{M_{5}}^{1}-\gamma_{H_{3}}^{2}-\gamma_{H_{1}}^{1}\right) \\
{\left[\mathbf{T}_{T Z_{1}, P_{6 B}}\right]_{\mathbf{y}}=} & -v_{H_{1}}^{1}+v_{M_{5}}^{1}+v_{H_{3}}^{2}-v_{M_{6}}^{2}+5 \gamma_{M_{6}}^{2} \\
& +37.5 \cdot\left(\gamma_{M_{5}}^{1}-\gamma_{H_{3}}^{2}-\gamma_{H_{1}}^{1}\right)
\end{aligned}
$$

Note that deviations of part-holder surfaces $\mathrm{H}_{2}$ and $\mathrm{H}_{4}$ do not influence on this KPC.

- $K P C_{2}$ : the deviation of $K P C_{2}$ is defined as

$K P C_{2}=\max \left(\left|\left[\mathbf{T}_{T Z_{2}, P_{7 A}}\right]_{\mathbf{y}}\right|,\left|\left[\mathbf{T}_{T Z_{2}, P_{7 B}}\right]_{\mathbf{y}}\right|\right)$,

where

$$
\begin{aligned}
{\left[\mathbf{T}_{T Z_{2}, P_{7 A}}\right]_{\mathbf{y}}=} & -v_{H_{4}}^{2}-v_{M_{7}}^{2}-10 \gamma_{H_{4}}^{2}-2.5 \gamma_{M_{7}}^{2} \\
& -25 \cdot\left(\gamma_{M_{5}}^{1}-\gamma_{H_{3}}^{2}-\gamma_{H_{1}}^{1}\right), \\
{\left[\mathbf{T}_{T Z_{2}, P_{7 B}}\right]_{\mathbf{y}}=} & -v_{H_{4}}^{2}-v_{M_{7}}^{2}-10 \gamma_{H_{4}}^{2}+2.5 \gamma_{M_{7}}^{2} \\
& -20 \cdot\left(\gamma_{M_{5}}^{1}-\gamma_{H_{3}}^{2}-\gamma_{H_{1}}^{1}\right) .
\end{aligned}
$$

In this case, note that deviations of the part-holder surface $\mathrm{H}_{2}$ do not influence on this KPC. Furthermore, the machining deviations of surface $S_{6}$ do not either influence.

- $K P C_{3}$ : the deviation of $K P C_{3}$ is defined as

$K P C_{3}=\left|\left[\mathbf{T}_{T Z_{1}, P_{6 A}}\right]_{\mathbf{y}}-\left[\mathbf{T}_{T Z_{1}, P_{6 B}}\right]_{\mathbf{y}}\right|$,

where $\left[\mathbf{T}_{T Z_{1}, P_{6 A}}\right]_{\mathbf{y}}$ and $\left[\mathbf{T}_{T Z_{1}, P_{6 B}}\right]_{\mathbf{y}}$ are defined by Eq. (44) and (45) respectively. By substituting, the deviation is defined as $\mid 10 \gamma_{M_{5}}^{1}+10 \gamma_{M_{6}}^{2}-10 \gamma_{H_{3}}^{2}-$ $10 \gamma_{H_{1}}^{1} \mid$. Similar to the example shown previously, as this KPC is a parallelism relationship, translational machining deviations in any station do not influence. Furthermore, deviations of part-holder surfaces $H_{1}$ and $H_{3}$ in $X$ or $Y$ direction do not influence, and only their orientation deviation influences on the parallelism relationship.

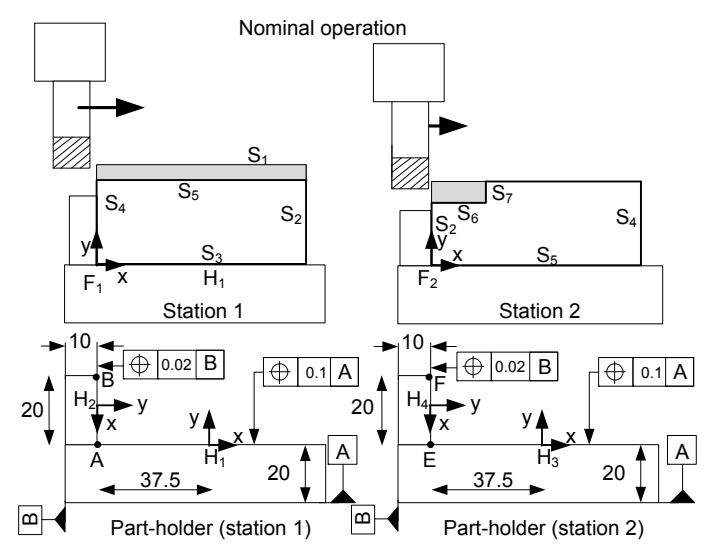

Fig. 7 Multi-station machining process to manufacture the 2D example. Dimensions in $\mathrm{mm}$.

Note that if Eqs. (39) and (40) do not apply, it means that the workpiece-fixture assembly is different than the configurations analyzed and the resulting positioning deviation torsor varies. To analyze those cases, Eqs.(41) and (42) should be re-evaluated considering the points $A$ and $E$ as contact points between workpiece and part-holder at station 1 and 2 respectively.

\subsubsection{Numerical resolution}

Similar to the SoV case study, the case study of the MoMP is numerically solved analyzing the worst-case and the statistical approach. The variability range for each manufacturing process variable is shown in Table 9. Unlike the SoV case study, where the locators variability is independent to each other, in the MoMP the variability of a surface depends on different torsor parameters and those parameters are not independent to each other since they are subjected to the geometri$\mathrm{cal} /$ dimensional tolerance of the surface. For this case study, part-holder surfaces are planar and a position tolerance zone applies for each one.

The numerical resolution for worst-case analysis requires a search algorithm. In this case study it is applied two algorithms sequentially. Firstly, a genetic algorithm (GA) in order to find a region close to the optimal solution. Secondly, the solution provided by the GA is used as the initial point in a mesh adaptative direct search (MADS) algorithm for tuning the optimal result. The resolution was repeated 10 times to ensure the optimization convergence, so the global minimum reached provides the worst-case solution. The numerical resolution for the statistical analysis requires the evaluation of thousands of samples through Monte Carlo simulations. For this case study, 5,000 Monte Carlo simulations were run assuming that all torsor parameters 
Table 9 Precision of part-holder surfaces and machined surfaces for the MoMP case study. Dimensional deviations in -mm-, angular deviations in -rad-.

\begin{tabular}{l} 
Station 1 \\
\hline Precision of part-holder surface $1: t=0.1 \mathrm{~mm}$; torsor constraints: $-\frac{0.1}{2} \leq v_{H_{1}}^{1}+37.5 \cdot \gamma_{H_{1}}^{1} \leq \frac{0.1}{2}$ \\
Precision of part-holder surface $2: t=0.02 \mathrm{~mm}$; torsor constraints: $-\frac{0.02}{2} \leq v_{H_{2}}^{1}+10 \cdot \gamma_{H_{2}}^{1} \leq \frac{0.02}{2}$ \\
Machining precision: $\quad-0.01 \leq u_{M_{5}}^{1} \leq 0.01 \quad-0.01 \leq v_{M_{5}}^{1} \leq 0.01 \quad-0.001 \leq \gamma_{M_{5}}^{1} \leq 0.001$ \\
\hline Station 2 \\
\hline Precision of part-holder surface $3: t=0.1 \mathrm{~mm}$; torsor constraints: $-\frac{0.1}{2} \leq v_{H_{3}}^{2}+37.5 \cdot \gamma_{H_{3}}^{2} \leq \frac{0.1}{2}$ \\
Precision of part-holder surface $4: t=0.02 \mathrm{~mm}$; torsor constraints: $-\frac{0.02}{2} \leq v_{H_{4}}^{2}+10 \cdot \gamma_{H_{4}}^{2} \leq \frac{0.02}{2}$ \\
Machining precision: $\quad-0.01 \leq u_{M_{6}}^{2} \leq 0.01 \quad-0.01 \leq v_{M_{6}}^{2} \leq 0.01 \quad-0.001 \leq \gamma_{M_{6}}^{2} \leq 0.001$ \\
Machining precision: $\quad-0.01 \leq u_{M_{7}}^{2} \leq 0.01 \quad-0.01 \leq v_{M_{7}}^{2} \leq 0.01 \quad-0.001 \leq \gamma_{M_{7}}^{2} \leq 0.001$
\end{tabular}

Table 10 Numerical resolution according to the worst-case (WC) and the statistical (ST) analysis for the MoMP case study. Dimensions in $-m m-$.

\begin{tabular}{l|ccc} 
& $K P C_{1}$ & $K P C_{2}$ & $K P C_{3}$ \\
\hline WC (Pro/E) & \pm 0.163 & \pm 0.114 & \pm 0.047 \\
WC & \pm 0.162 & \pm 0.114 & \pm 0.047 \\
ST (6 $\sigma$ interval) & \pm 0.091 & \pm 0.037 & \pm 0.018 \\
\hline
\end{tabular}

are normally distributed with the $6 \sigma$ ranges shown in Table 9 subjected to their tolerance constraints. In order to validate the model, the same worst-case analysis using Pro/Engineer Wildfire 5.0 as explained in subsection 4.1.2 was conducted. Table 10 shows the KPC variations according to the type of analysis conducted.

\section{Comparison and discussion}

\subsection{Fixture and processes supported}

The SoV model is based on a linear system of equations described in a matricial form in a similar way as the well-know state space model from control theory. For this reason, the SoV model has been used for modeling isostatic fixtures based on punctual locators according to the 3-2-1 layout. Hyperstatic fixtures based on locators or other workholding devices such as vises or 4-jaws chucks have not been considered. The MoMP overcomes this limitation since the formulation lets consider nonpunctual contacts for modeling the fixture assembly. Therefore, hyperstatic fixtures with a defined hierarchy of contacts, and industrial fixtures such as vises or chucks can be derived.

In terms of machining systems, the SoV model deals only with milling operations since in this process all degrees of freedom are constrained, and the applica- tion of the SoV model in its matricial form for diagnosis and process improvement is straightforward. The MoMP has been derived for both milling and turning processes, making it more applicable in real MMPs.

\subsection{Limitations of virtual inspection}

At the virtual inspection station, the SoV model conducts a point-based inspection similar to that conducted by a coordinate measuring machine (CMM) including in the model the measurement error in the inspection process. The virtual inspection and verification can be conducted straightforward for the worst-case and statistical analysis by evaluating one single expression (without conducting a large number of simulations or using an optimization algorithm). In the case of the MoMP, the deviation of the inspected surfaces is analyzed through a virtual gauge, so the inspection is a surface-based process where the precision of the gauge system can be considered. According to the tolerance accumulation, the MoMP can conduct both worst-case and statistical analysis. However, the worst-case analysis requires the resolution of a complex optimization problem where the global minimum solution (and thus, the real worstcase solution) may not be reached. On the other hand, the statistical analysis requires time-consuming Monte Carlo simulations to infer, from thousands of simulated samples, the statistical results of the virtual inspection. Thus, the resolution of both worst-case and statistical analysis are quite complex in comparison with the same analysis conducted under the SoV model. This is because the SoV model includes the individual source of errors in the manufacturing process (e.g. individual locator errors in isostatic fixture devices) which are independent to each other, whereas the MoMP models the deviations of surfaces by the SDT whose parameters 
are not independent. In other words, the SoV model is applied only in simple isostatic fixture configurations based on locators that greatly facilitates both worstcase and statistical analysis. For more complex fixtures, the SoV cannot be applied and the resolution should be conducted applying the MoMP solving a complex optimization problem for both worst-case and statistical cases.

\subsection{GD\&T conformance}

The SoV model virtually inspects the parts as they were inspected in a CMM. Therefore, the SoV model is oriented to vectorial dimensioning and tolerancing (VD\&T) specifications and common product design specifications based on GD\&T should be translated in order to be applied correctly. The mathematical translation of some GD\&T specifications such as parallelism, angularity and perpendicularity to be used by the SoV was investigated in [53]. Kong et al. [54] also included into the SoV model other GD\&T specifications such as material condition modifiers.

Unlike SoV model, the MoMP describes surface deviations using SDTs and uses gauges for inspection. These two characteristics make the MoMP more friendly to deal with GD\&T specifications than the SoV model. For instance, the MoMP has been successfully applied for manufacturing tolerancing according to ISO standards and GD\&T specifications [49]. The MoMP has been also applied to deal with material condition modifiers and incomplete datum frames $[39,42]$.

\subsection{Applicability}

The state space model formulation in the SoV model makes it to be preferred to the MoMP model when the applicability of the model is mainly focused on part quality improvement activities such as fault diagnosis, process diagnosability analysis, dimensional quality control and fixture maintenance planning. However, this applicability can be only conducted in milling processes with isostatic fixtures. In this field, the formulation of the MoMP makes it more difficult to be applied since there is no single matrix equation to be straighforward analyzed as there is in the SoV model.

Unlike the SoV model, the MoMP is oriented to product design activities. This is mainly because of the ability of SDTs to model surface tolerances and because of the contact between surfaces is not only based on punctual contacts but also on surface contacts.
5.5 Model accuracy

Considering the modeling mechanism, both SoV and MoMP models are based on kinematic chains and homogeneous transformation matrices. Both assume solid rigid parts and they can not deal with form errors (intrinsic part tolerances), so they are focused on position and orientation errors (extrinsic part tolerances). In manufacturing context, angular deviations due to manufacturing error remain very small, so the general matrix approach con be simplified using SDT or DMV [55]. Simulations conducted with Pro/Engineer are shown in Tables 6 and 10, and they reveal that, for the worst case analysis, the estimation error of KPCs is below $1 \mu \mathrm{m}$ for both approaches.

It should be noted that both models require a comprehensive information of the product and the manufacturing process such as geometry of nominal part surfaces, manufacturing and fixture capabilities, geometrical information of fixture configurations, placement of the inspection stations, capability of the inspection systems, etc. The large quantity of a priori engineering knowledge required and its level of accuracy has an important impact on the final accuracy of the model, which might prevent their application in largescale MMPs [56]. However, for low and medium-scale systems the application of SoV and MoMP models is highly recommended to eliminate downstream manufacturing problems and reduce ramp-up times.

\subsection{Modeling complexity}

The derivation of the SoV model is, in authors' opinion, more oriented to multi-station systems than the MoMP due to the adoption of the state space model of control theory, and it is more easily to be automated due to its matrix formulation. The multi-station representation by the SoV model is clearer than that from the MoMP model, and the inclusion of the inspection stations is also straightforward. Furthermore, the resolution of worst-case and statistical analysis are also easier and faster since it requires only the evaluation of a system of equations in matrix form. However, it should be noted that there are more limitations applied in the SoV model, so complex MMPs based on turning-milling processes and non-punctual isostatic fixtures should require the application of the MoMP. 


\section{Conclusion and future work}

The development of 3D manufacturing variation models for integrating product and manufacturing process design is essential to improve product development, eliminate downstream manufacturing problems and reduce ramp-up times. In the literature, two main 3D manufacturing variation models in MMPs have been studied: the Stream of Variation (SoV) model and the Model of the Manufactured Part (MoMP). This paper has presented both SoV and MoMP models, analyzing and comparing their main characteristics and applications. Furthermore, the methodology to derive both models has been described step by step by a simple $2 \mathrm{D}$ case study (extensible for any 3D part). As future work, some potential improvements on manufacturing variation propagation models in MMPs should be conducted. As some ideas, the authors suggest:

- Both SoV and MoMP models are restricted to solid rigid components. Industrial machining operations also require the analysis of manufacturing variability on complaint parts due to deformations induced by clamp forces or cutting-tool forces.

- Both SoV and MoMP models do not include form errors. Although form errors are considered to be not as critical as dimension or orientation errors for 3D manufacturing variation, research efforts should be conducted to include in somehow these geometrical variations.

- SoV models deal with fixtures composed of locators, but their extension to cover common industrial fixtures such as vises or chucks should be addressed.

- SoV models can deal with non-orthogonal 3-2-1 fixture layouts but common hyperstatic fixture schemes in industry such as N-2-1 layouts are still unaddressed.

- The derivation of the MoMP includes generic machinetools capabilities by defining different torsor components. However, machine-tool capabilities can be expressed by their sources of variation themselves such as cutting-tool deflections, tooling wear, etc. The inclusion of additional chain of torsors to explicitly indicate the effect of these machining variations could be interesting for future applications in manufacturing tolerancing.

- The application of the MoMP for process tolerance allocation is still unaddressed. This process tolerance allocation should be analyzed by integrating the different cost functions of the manufacturing process such as fixture maintenance operations or cutting-tool replacement policies among others.
Acknowledgements This work has been partially supported by Fundació Caixa-Castelló Bancaixa (Research Promotion 2007, 2009 and 2011).

\section{References}

1. Ceglarek D, Huang W, Zhou S, Ding Y, Kumar R, Zhou Y (2004) Time-based competition in multistage manufacturing: Stream-of-variation analysis (SOVA) methodology-review. International Journal of Flexible Manufacturing Systems 16:11-44.

2. Shi J (2007) Stream of variation modeling and analysis for multistage manufacturing systems. CRC Press Taylor and Francis Group.

3. Ogata K (2001) Modern control engineering. Prentice Hall, 4th edition.

4. Zhou S, Huang Q, Shi J (2003) State space modeling of dimensional variation propagation in multistage machining process using differential motion vectors. IEEE Transactions on Robotics and Automation 19:296-309.

5. Huang Q, Shi J, Yuan J (2003) Part dimensional error and its propagation modeling in multi-operational machining processes. Journal of Manufacturing Science and Engineering 125:255-262.

6. Djurdjanovic D, Ni J (2003) Dimensional errors of fixtures, locating and measurement datum features in the stream of variation modeling in machining. Journal of Manufacturing Science and Engineering-Transactions of the ASMe 125:716-730.

7. Loose JP, Zhou S, Ceglarek D (2007) Kinematic analysis of dimensional variation propagation for multistage machining processes with general fixture layouts. IEEE Transactions on Automation Science and Engineering 4:141-152.

8. Abellan-Nebot JV, Liu J, Romero F (2012) State space modeling of variation propagation in multi-station machining processes considering machining-induced variations. Journal of Manufacturing Science and Engineering In Press

9. Zhang M, Djurdjanovic D, Ni J (2007) Diagnosibility and sensitivity analysis for multi-station machining processes. International Journal of Machine Tools and Manufacture 47:646-657.

10. Liu J, Shi J, Hu SJ (2009) Quality-assured setup planning based on the stream-of-variation model for multi-stage machining processes. IIE Transactions 41:323-334(12).

11. Abellan-Nebot JV, Liu J, Subiron FR (2011) Design of multi-station manufacturing processes by integrating the stream-of-variation model and shop-floor data. Journal of Manufacturing Systems 30:70 - 82.

12. Wang H, Huang Q, Katz R (2005) Multi-operational machining processes modeling for sequential root cause identification and measurement reduction. Journal of Manufacturing Science and Engineering 127:512-521.

13. Ding Y, Shi J, Ceglarek D (2002) Diagnosability analysis of multi-station manufacturing processes. Journal of Dynamic Systems, Measurement, and Control 124:1-13.

14. Zhou S, Chen Y, Ding Y, Shi J (2003) Diagnosability study of multistage manufacturing processes based on linear mixed-effects models. Technometrics 45:312-325.

15. Zhou SY, Chen Y, Shi J (2004) Statistical estimation and testing for variation root-cause identification of multistage manufacturing processes. IEEE Transactions on Automation Science and Engineering 1:73-83. 
16. Li ZG, Zhou SY, Ding Y (2007) Pattern matching for variation-source identification in manufacturing processes in the presence of unstructured noise. IIE Transactions 39:251-263.

17. Djurdjanovic D, Ni J (2003) Bayesian approach to measurement scheme analysis in multistation machining systems. Proceedings of the Institution of Mechanical Engineers Part B-Journal of Engineering Manufacture 217:1117-1130.

18. Ding Y, Kim P, Ceglarek D, Jin J (2003) Optimal sensor distribution for variation diagnosis in multistation assembly processes. IEEE Transactions on Robotics and Automation 19:543-556.

19. Djurdjanovic D, Ni J (2004) Measurement scheme synthesis in multi-station machining systems. Journal of Manufacturing Science and Engineering 126:178-188.

20. Djurdjanovic D, Ni J (2006) Stream-of-variation (SoV)based measurement scheme analysis in multistation machining systems. IEEE Transactions on Automation Science and Engineering 3:407-422.

21. Izquierdo L, Shi J, Hu S, Wampler C (2007) Feedforward control of multistage assembly processes using programmable tooling. NAMRI/SME Transactions 35:295302.

22. Djurdjanovic D, Zhu J (2005) Stream of variation based error compensation strategy in multi-stage manufacturing processes. ASME Conference Proceedings .

23. Djurdjanovic D, Ni J (2007) Online stochastic control of dimensional quality in multistation manufacturing systems. Proceedings of the Institution of Mechanical Engineers Part B-Journal of Engineering Manufacture 221:865-880.

24. Jiao Y, Djurdjanovic D (2008) Allocation of flexible tooling for optimal stochastic multistation manufacturing process quality control. ASME Conference Proceedings 2008:161-169.

25. Jiao Y, Djurdjanovic D (2010) Joint allocation of measurement points and controllable tooling machines in multistage manufacturing processes. IIE Transactions 42:703-720.

26. Abellan-Nebot JV, Liu J, Subiron FR (2012) Quality prediction and compensation in multi-station machining processes using sensor-based fixtures. Robotics and Computer-Integrated Manufacturing 28:208 - 219.

27. Chen Y, Ding Y, Jin J, Ceglarek D (2006) Integration of process-oriented tolerancing and maintenance planning in design of multistation manufacturing processes. IEEE Transactions on Automation Science and Engineering 3:440-453.

28. Ding Y, Jin JH, Ceglarek D, Shi J (2005) Processoriented tolerancing for multi-station assembly systems. IIE Transactions 37:493-508.

29. Shirinzadeh B (2002) Flexible fixturing for workpiece positioning and constraining. Assembly Automation 22:112-120.

30. Ding Y, Ceglarek D, Jin JH, Shi JJ (2000) Processoriented tolerance synthesis for multistage manufacturing systems. In Proceedings of the International Mechanical Engineering Congress and Exposition, Orlando, FL, pp $15-22$.

31. Ballot E, Bourdet P (1997) A computational method for the consequences of geometric errors in mechanisms. In Proceedings of the 4th CIRP Seminar on Computer Aided Tolerancing, Tokyo, Japan, pp 137-148.

32. Chase KW, Gao J, Magleby SP (1995) General 2-D tolerance analysis of mechanical assemblies with small kinematic adjustments. Journal of Design and Manufacturing 5.
33. Chase KW, Gao J, Magleby SP, Sorensen CD (1996) Including geometric feature variations in tolerance analysis of mechanical assemblies. IIE Transactions 28:795-807.

34. Davidson JK, Mujezinovic A, Shah JJ (2002) A new mathematical model for geometric tolerances as applied to round faces. Journal of Mechanical Design 124:609622 .

35. Villeneuve F, Legoff O, Landon Y (2001) Tolerancing for manufacturing: a three-dimensional model. International Journal of Production Research 39:1625-1648.

36. Desrochers A (1999) Modeling three-dimensional tolerance zones using screw parameters. In CD-ROM Proceedings of ASME 25th Design Automation Conference, DAC-8587, Las-Vegas.

37. Desrochers A, Ghie W, Laperriere L (2003) Application of a unified jacobian-torsor model for tolerance analysis. Journal of Computing and Information Science in Engineering 3:2-14.

38. Villeneuve F, Vignat F (2007) Simulation of the manufacturing process in a tolerancing point of view: Generic resolution of the positioning problem. In Models for Computer Aided Tolerancing in Design and Manufacturing, pp 179-189.

39. Vignat F, Villeneuve F (2003) 3D transfer of tolerances using a SDT approach: Application to turning process. Journal of Computing and Information Science in Engineering 3:45-53.

40. Nejad MK, Vignat F, Desrochers A, Villeneuve F (2010) 3D simulation of manufacturing defects for tolerance analysis. Journal of Computing and Information Science in Engineering 10:021001.

41. Ghie W, Laperrire L, Desrochers A (2007) Re-design of mechanical assemblies using the unified jacobian-torsor model for tolerance analysis. In Models for Computer Aided Tolerancing in Design and Manufacturing, pp 95104.

42. Nejad MK, Vignat F, Villeneuve F (2009) Simulation of the geometrical defects of manufacturing. The International Journal of Advanced Manufacturing Technology 45:631-648.

43. Villeneuve F, Vignat F (2005) Manufacturing process simulation for tolerance analysis and synthesis. In Advances in Integrated Design and Manufacturing in Mechanical Engineering, pp 189-200.

44. Nejad MK (2010) Propositions de résolution numérique des problèmes d'analyse de tolérance en fabrication : approche 3D, Dissertation, Université Joseph Fourier.

45. Ayadi B, Anselmetti B, Bouaziz Z, Zghal A (2008) Threedimensional modelling of manufacturing tolerancing using the ascendant approach. The International Journal of Advanced Manufacturing Technology 39:279-290.

46. Louati J, Ayadi B, Bouaziz Z, Haddar M (2006) Threedimensional modelling of geometric defaults to optimize a manufactured part setting. The International Journal of Advanced Manufacturing Technology 29:342-348.

47. Tichadou S, Legoff O, Hascoet JY (2005) 3D geometrical manufacturing simulation. In Advances in Integrated Design and Manufacturing in Mechanical Engineering, pp 201-214.

48. Kamali Nejad M, Vignat F, Villeneuve F (2012) Tolerance analysis in machining using the model of manufactured part (MMP) - comparison and evaluation of three different approaches. International Journal of Computer Integrated Manufacturing 25:136-149.

49. Anselmetti B, Louati H (2005) Generation of manufacturing tolerancing with ISO standards. International 
Journal of Machine Tools and Manufacture 45:1124 1131.

50. Vignat F, Villeneuve F (2007) A numerical approach for 3D manufacturing tolerances synthesis. In 10th CIRP Conference on Computer Aided Tolerancing, Erlangen, Germany.

51. Tichadou S, Kamalinejad M, Vignat F, Legoff O (2007) 3-d manufacturing dispersions: Two experimental applications. In 10th CIRP International Conference on Computer Aided Tolerancing, Erlangen, Germany.

52. Sergent A, Bui MH, Favreliere H, Duret D, Samper S, Villeneuve F (2010) Identification of machining defects by small displacement torsor and form parameterization method. In IDMME International Conference, Bordeaux, France.

53. Loose J, Zhou Q, Zhou S, Ceglarek D (2010) Integrating GD\&T into dimensional variation models for multistage machining processes. International Journal of Production Research 48:3129-3149.

54. Kong Z, Huang W, Oztekin A (2009) Variation propagation analysis for multistation assembly process with consideration of GD\&T factors. Journal of Manufacturing Science and Engineering 131:051010.

55. Bourdet P, Ballot E (1995) Geometrical behaviour laws for computer aided tolerancing. In 4th CIRP Seminars on Computer Aided Tolerancing, Tokyo, pp 143-154.

56. Liu J, Shi J, Hu SJ (2008) Engineering-driven factor analysis for variation source identification in multistage manufacturing processes. Journal of Manufacturing Science and Engineering 130:041009. 